\title{
ANÁLISE BAYESIANA DE MODELOS LINEARES MISTOS ROBUSTOS VIA AMOSTRADOR DE GIBBS
}

\author{
GUILHERME JORDĀO DE MAGALHÃES ROSA \\ Zootecnista
}

Orientador: Prof. Dr. CARLOS ROBERTO PADOVANI

Tese apresentada à Escola Superior de Agricultura "Luiz de Queiroz", da Universidade de São Paulo, para obtenção do título de Doutor em Agronomia, Área de Concentração: Estatística e Experimentação Agronômica.

P I R A C I C A B A

Estado de São Paulo - Brasil

Dezembro - 1998 


\section{Dados Internacionais de Catalogação na Publicação (CIP) DIVISÃO DE BIBLIOTECA E DOCUMENTAÇÃO - Campus "Luiz de Queiroz"/USP}

Rosa, Guilherme Jordão de Magalhães

Análise bayesiana de modelos lineares mistos robustos via Amostrador de Gibbs /

Guilherme Jordăo de Magalhāes Rosa. - Piracicaba, 1998.

$57 \mathrm{p}$.

Tese (doutorado) - Escola Superior de Agricultura Luiz de Queiroz, 1998.

Bibliografia.

1. Amostragem 2. Distribuiçăo 3. Estatistica aplicada 4. Método de Bayes 5. Modelo matemático I. Título

CDD 519.532 
À minha mae, sempre companheira e amiga, com eterna gratidão, dedico com carinho e amor. 


\section{Agradecimentos}

Ao Prof. Dr. Carlos Roberto Padovani, a orientação, a amizade e o constante estímulo.

Ao Prof. Dr. Daniel Gianola, a supervisão e todas as facilidades oferecidas durante o período de estudos na "University of Wisconsin - Madison".

À Fundação Coordenação de Aperfeiçoamento de Pessoal de Nível Superior (CAPES) a bolsa de estudos.

Aos companheiros do Departamento de Bioestatística - IB, UNESP, Botucatu, todo o apoio.

Aos Professores e funcionários do Departamento de Matemática e Estatística da ESALQ/USP, a atenção e a amizade.

Ao Tom, Peter, Jorge e Cristián pelo auxílio computacional prestado.

À minha tia Naisa, o incentivo e a ajuda constantes.

Aos colegas de curso, Chico, Ogliari, Paulo, Sérgio e Sílvia, o companheirismo e a amizade.

Aos colegas em Madison, Brett, Guo, Marcelo, Men, Parag, Ruby e, em especial, à Natalia, os momentos compartilhados.

$\grave{A}$ todos os demais que, direta ou indiretamente, colaboraram nesta etapa da minha vida. 


\section{SUMÁRIO}

Página

Lista DE FigURAS

$$
\mathrm{v}
$$

LISTA DE TABELAS

vi

RESUMO

vii

SUMMARY viii

1 INTRODUÇÃO

2 REVISĀo DE LITERATURA 3

2.1 Modelos lineares mistos . . . . . . . . . . . . . . . . 3

2.1 .1 Definição e notação geral . . . . . . . . . . . . . 3

2.1.2 Estimação de máxima verossimilhança para $\boldsymbol{\beta}$ e $\mathbf{b} \ldots \ldots . .44$

2.1.3 Estimação dos componentes de variância . . . . . . . . . . 5

2.2 Inferência bayesiana . . . . . . . . . . . . . . . 6

2.3 Amostrador de Gibbs . . . . . . . . . . . . . . . . . . . . . . . . 8

2.4 Distribuições normal/independentes . . . . . . . . . . . . . . . . . . . . . 99

2.4 .1 Definição . . . . . . . . . . . . . . . . . . . 9

2.4 .2 Algumas propriedades . . . . . . . . . . . . . . 10

2.4 .3 Distribuição $t$ de Student . . . . . . . . . . . . . . . . . 14

2.4 .4 Distribuição slash . . . . . . . . . . . . . . . . 16

2.4 .5 Distribuição normal contaminada . . . . . . . . . . 16

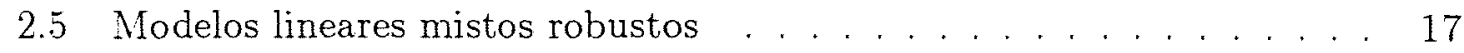

3 METODOLOGIA $\quad 19$

3.1 Modelo amostral e função de verossimilhança . . . . . . . . . . 20

3.2 Distribuições a priori e densidade conjunta a posteriori . . . . . . 20 
3.3 Distribuições condicionais completas a posteriori . . . . . . . . . . 22

3.4 Exemplos com distribuições normal/independentes . . . . . . . . . . . 23

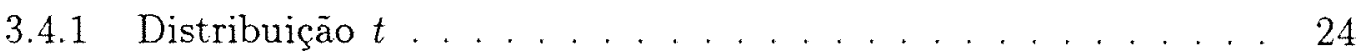

3.4 .2 Distribuição slash . . . . . . . . . . . . . . . . . 25

3.4 .3 Distribuição normal contaminada . . . . . . . . . . . . . . 26

4 EXEMPLOS DE APLICAÇÃO 30

4.1 Estudo reprodutivo em ratas . . . . . . . . . . . . . . . . . . 30

4.1.1 Procedimentos metodológicos . . . . . . . . . . . . . . 30

4.1.2 Resultados e discussão . . . . . . . . . . . . . . . . 33

4.2 Estudo ortodôntico em crianças . . . . . . . . . . . . . . . . . 39

4.2.1 Procedimentos metodológicos . . . . . . . . . . . . . . 39

4.2 .2 Resultados e discussão . . . . . . . . . . . . . . . 42

5 CONSIDERAÇŌES FINAIS $\quad 49$

REFERENNCIAS BIBLIOGRÄFICAS 


\section{Lista DE FIGURAS}

Página

1 Resíduos padronizados de cada indivíduo, em cada ninhada, para o modelo gaussiano (Os números 1,2 e 3 denotam animais que serão utilizados em discussão posterior com relação à detecção de valores discrepantes). . . . . . . . . . . . . . . . . 32

2 Densidade marginal a posteriori $\operatorname{dos}$ parâmetros $\nu$ de cada distribuição considerada, para as situações multivariadas (linhas segmentadas) e univariadas (linhas contínuas) . . . . . . . . . . . . . . . 36

3 Gráfico "box plot" dos valores obtidos pelo Amostrador de Gibbs para o contraste entre pesos de machos e fêmeas, para cada um dos modelos considerados (n: normal: $\mathrm{m}$ : multivariada; $\mathrm{u}$ : univariada; $\mathrm{t}$ : $t$ de Student; s: slash; e c: normal contaminada).

4 Distribuições marginais a posteriori das variáveis ponderadoras dos indivíduos 1, 2 e 3 assinalados na Figura 1, para as distribuições nor$\mathrm{mal} /$ independentes consideradas $(\mathrm{O}$ eixo adicional à direita refere-se à probabilidade de $w_{i j}=1$ ).

5 Regressões de mínimos quadrados da distância $(\mathrm{mm})$ entre a pituitária e a fissura pterigomaxilar em função da idade (anos), para os 16 meninos e 11 meninas do estudo ortodôntico (Adaptado de Pinheiro et al. (1998)).

6 Densidades marginais a posteriori estimadas para cada elemento do vetor de efeitos "fixos" relativo ao estudo ortodôntico com crianças, considerando-se o modelo Normal 3 (linhas segmentadas) e o modelo Robusto 4 (linhas contínuas). 


\section{Lista DE TABELAS}

Página

1 Estimativas dos componentes de variância e de alguns contrastes e parâmetros de interesse para o conjunto de dados de peso ao nascimento de ratos. . . . . . . . . . . . . . . . . . . . . . 31

2 Média, mediana, desvio padrão (DP) e percentis $(2,5$ e $97,5 \%)$ das distribuições a posteriori marginais de alguns contrastes e parâmetros de interesse para o conjunto de dados de peso ao nascimento de ratos. 34

3 Densidade de probabilidade marginal dos dados para cada um dos modelos considerados, $p(y \mid$ Modelo $)$.

4 Caracterização dos modelos lineares gaussianos utilizados no estudo ortodôntico em crianças.

5 Densidade de probabilidade marginal dos dados para cada um dos modelos gaussianos considerados.

6 Densidade de probabilidade marginal dos dados para cada um dos modelos $t$ considerados. 


\title{
ANÁlISE BAYESIANA DE MODELOS LINEARES MISTOS ROBUSTOS VIA AMOSTRADOR DE GIBBS
}

\author{
Autor: GUILHERME JORDÃO DE MAGALHĀES ROSA \\ Orientador: Prof. Dr. CARLOS ROBERTO PADOVANI
}

\section{RESUMO}

Os modelos lineares de efeitos mistos têm sido amplamente utilizados na análise de dados onde as respostas estão agrupadas e a suposição de independência entre observações num mesmo grupo não é adequada.

$\mathrm{Na}$ maioria das aplicações desses modelos é assumida distribuição normal tanto dos resíduos quanto dos efeitos aleatórios, o que os tornam muito sensíveis à presença de valores discrepantes nas observações.

No presente trabalho é discutida a utilização de um grupo de distribuições leptocúrticas, denominadas distribuições normal/independentes, como alternativas robustas para a distribuição gaussiana.

Toda a apresentação é efetuada dentro de uma perspectiva bayesiana e o Amostrador de Gibbs é utilizado na condução da análise a posteriori.

Dois exemplos de aplicações utilizando-se a distribuição $t$, a distribuição slash e a distribuição normal contaminada são apresentados para ilustrar a metodologia.

As distribuições normal/independentes mostram-se como alternativas robustas bastante interessantes para modelos lineares mistos, sendo de fácil implementação dentro de um contexto bayesiano, podendo ser também utilizadas na detecção de valores discrepantes em conjuntos de dados. 


\title{
BAYESIAN ANALYSIS OF ROBUST LINEAR MIXED MODELS VIA GIBBS SAMPLING
}

\author{
Author: GUILHERME JORDÃO DE MAGALHÃES ROSA \\ Adviser: Prof. Dr. CARLOS ROBERTO PADOVANI
}

\section{SUMMARY}

Linear mixed models have been used widely in analysis of data where the responses are clustered, so it is not possible to assume independence between observations in the same cluster.

In most applications of these models, it is assumed that the distribution of the residuals and of the random effects is Gaussian; this makes the model vulnerable to the presence of outliers.

Some long-tailed distributions, called generically normal/independent distributions, are studied here as robust alternatives to the normal distribution, in the context of mixed effects models.

The developments are presented from a Bayesian perspective, and the Gibbs sampler is used to implement the posterior analysis.

Two real examples using the Student- $t$, the slash and the contaminated normal distributions are presented to illustrate the methodology.

The normal/independent distributions are found to be interesting robust alternatives to the Gaussian in linear mixed models, and are easy to be applied within a Bayesian context. The developments allow to use the model output as an instrument for outlier detection. 


\section{INTRODUÇÃO}

Os modelos lineares de efeitos mistos, ou simplesmente modelos lineares mistos (Laird \& Ware, 1982; Lindstrom \& Bates, 1988), têm sido amplamente utilizados na análise de dados onde as respostas estão agrupadas e a suposição de independência entre observações num mesmo grupo não é adequada. Exemplos deste tipo de situação podem ser encontrados em medidas repetidas em estudos longitudinais ou em membros de uma mesma família em estudos genéticos (Zeger \& Karin, 1991).

Na maioria das aplicações dos modelos lineares mistos é assumida distribuição normal tanto dos resíduos quanto dos efeitos aleatórios, o que os tornam muito sensíveis à presença de valores discrepantes nas observações (Rogers \& Tukey, 1972).

A utilização de modelos com distribuições leptocúrticas em substituição à distribuição normal tem-se mostrado bastante eficiente e de fácil implementação para estimação robusta em análise de dados em diversas situações.

A mais conhecida distribuição utilizada com esse objetivo é a distribuição $t$ de Student, dada a sua simplicidade e o fato de sua robustez ser definida por somente um único parâmetro, o número de graus de liberdade (Lange et al, 1989). Alguns exemplos de aplicações da distribuição $t$ em estimação robusta podem ser encontrados em Geweke (1993) e Liu \& Rubin (1995), entre outros.

Especificamente com modelos lineares mistos robustos encontram-se os trabalhos de Strandén (1996), dentro de um contexto bayesiano, e de Pinheiro et al. (1998), em estimação de máxima verossimilhança, ambos utilizando também a distribuição $t$ em suas apresentações.

Um grupo mais geral de distribuições leptocúrticas, do qual a distribuição té um caso particular, caracteriza-se pela mistura de distribuições normais, e são denominadas distribuições normal/independentes (Lange \& Sinsheimer, 1993). Este grupo de distribuições foi considerado por Liu (1996), que discute inferência bayesiana em modelos robustos de regressão. 
Objetiva-se no presente trabalho a discusão de modelos lineares mistos com distribuições normal/independentes tanto para resíduos quanto para os demais efeitos aleatórios dos mesmos. É adotado um procedimento bayesiano e o Amostrador de Gibbs é utilizado na condução da análise a posteriori. Dois exemplos de aplicação utilizando-se a distribuição $t$, a distribuição slash e a distribuição normal contaminada são apresentados para ilustrar a metodologia.

O primeiro conjunto de dados, apresentado por Dempster et al. (1984) e reanalisado por Davidian \& Giltinan (1995), refere-se ao peso ao nascimento de filhotes num estudo toxicológico em reprodução de ratas. Com esse exemplo, objetiva-se a comparação de algumas distribuições normal/independentes para os resíduos, bem como a comparação de modelos assumindo-se essas distribuiçōes como univariadas ou multivariadas para indivíduos sob mesmo grupo de efeitos aleatórios.

O segundo conjunto de dados utilizado foi apresentado inicialmente por Potthof \& Roy (1964), e mais recentemente rediscutido por Verbeke \& Molenberghs (1997) e por Pinheiro et al. (1998), e refere-se a um estudo ortodôntico com crianças com idades entre 8 e 14 anos. A variável resposta é a distância entre a pituitária e a fissura pterigomaxilar, a qual foi medida aos 8, 10, 12 e 14 anos de idade de cada indivíduo. Nesse exemplo foi adotada distribuição robusta também para os efeitos aleatórios do modelo e objetivou-se a utilização e comparação de diferentes modelos $t$ e gaussianos. 


\section{REVISÃO DE LITERATURA}

\subsection{Modelos lineares mistos}

\subsubsection{Definição e notação geral}

Modelos lineares mistos são modelos nos quais efeitos fixos e aleatórios contribuem linearmente para a variável resposta. Sua forma geral pode ser escrita como

$$
\mathrm{y}_{i}=X_{i} \boldsymbol{\beta}+Z_{i} \mathrm{~b}_{i}+\mathrm{e}_{i}
$$

onde $\mathrm{y}_{i}$ é o vetor de respostas de dimensão $n_{i}, i=1,2, \ldots, m, X_{i}$ e $Z_{i}$ são matrizes de delineamento (ou incidência) conhecidas, de dimensões $\left(n_{i} \times p\right)$ e $\left(n_{i} \times q\right)$, respectivamente; $\beta$ é um vetor $p$-dimensional contendo os efeitos fixos; $\mathbf{b}_{i}$ é um vetor $q$-dimensional contendo os efeitos aleatórios; e $\mathbf{e}_{i}$ é o vetor de resíduos, de dimensão $n_{i}$.

Em geral, assume-se que $b_{i}$ tem distribuição normal com média 0 e matriz de covariâncias $B$ não-singular, independente de $e_{i}$, o qual distribui-se normalmente com média 0 e matriz não-singular de covariâncias $\Sigma_{i}$, ou seja,

$$
\left[\begin{array}{c}
\mathbf{b}_{i} \\
\mathbf{e}_{i}
\end{array}\right] \stackrel{\text { ind. }}{\sim} N_{\left(q+n_{i}\right)}\left(\left[\begin{array}{l}
\mathbf{0} \\
\mathbf{0}
\end{array}\right],\left[\begin{array}{cc}
B & 0 \\
0 & \Sigma_{i}
\end{array}\right]\right) .
$$

Assim, tem-se que, condicionalmente ao vetor de efeitos aleatórios $\mathbf{b}_{i}$, $\mathrm{y}_{i}$ é normalmente distribuído com vetor de médias $X_{i} \boldsymbol{\beta}+Z_{i} \mathbf{b}_{i}$ e matriz de covariâncias $\Sigma_{i}$. A distribuição marginal de $\mathrm{y}_{i}$ é normal com vetor de médias $X_{i} \beta$ e matriz de covariâncias $V_{i}=Z_{i} B Z_{i}^{\prime}+\Sigma_{i}$.

Em geral, $\Sigma_{i}$ é definida por um pequeno número fixo de parâmetros. Um caso especial e bastante comum ocorre quando $\Sigma_{i}=\sigma^{2} I_{\left(n_{i}\right)}$, onde $I_{\left(n_{i}\right)}$ é uma matriz identidade de dimensão $n_{i}$.

Os modelos lineares de efeitos mistos para análise de variância (Miller, 1977), os modelos lineares para dados longitudinais (Laird \& Ware, 1982), os modelos de componentes de variância (Searle, et al., 1992), e mesmo modelos genéticos, tipo 
modelo animal ou modelo de reprodutor (Henderson, 1984), são todos casos especiais do modelo (1).

\subsubsection{Estimação de máxima verossimilhança para $\beta$ e b}

A função de máxima verossimilhança para $\beta$ e $b=\left[b_{1}^{\prime}, b_{2}^{\prime}, \ldots, b_{m}^{\prime}\right]^{\prime}$ pode ser escrita à semelhança da função de densidade de probabilidade conjunta de $\mathrm{y}=\left[\mathrm{y}_{1}^{\prime}, \mathrm{y}_{2}^{\prime}, \ldots, \mathrm{y}_{m}^{\prime}\right]^{\prime}$ e $\mathbf{b}$, ou seja

$$
\begin{aligned}
L(\boldsymbol{\beta}, \mathbf{b} \mid \mathbf{y}, B, \Sigma) & =p(\mathbf{y}, \mathbf{b} \mid \boldsymbol{\beta}, B, \Sigma) \\
& =p(\mathbf{y} \mid \boldsymbol{\beta}, \mathbf{b}, \Sigma) p(\mathbf{b} \mid B) \\
& \propto|\Sigma|^{-1 / 2} \exp \left\{-\frac{1}{2}(\mathbf{y}-X \boldsymbol{\beta}-Z \mathbf{b})^{\prime} \Sigma^{-1}(\mathbf{y}-X \boldsymbol{\beta}-Z \mathbf{b})\right\} \\
& \times|\mathbf{B}|^{-1 / 2} \exp \left\{-\frac{1}{2} \mathbf{b}^{\prime} \mathbf{B}^{-1} \mathbf{b}\right\},
\end{aligned}
$$

onde $\mathrm{X}=\left[X_{1}^{\prime}, X_{2}^{\prime}, \ldots, X_{m}^{\prime}\right]^{\prime}, \mathrm{Z}=D\left\{Z_{i}\right\}$, matriz bloco diagonal de dimensões $(n \times m q)$; $\Sigma=D\left\{\Sigma_{i}\right\}$, matriz bloco diagonal de dimensões $(n \times n)$, com $n=\sum_{i=1}^{m} n_{i}$; e $\mathbf{B}=D\{B\}$ matriz bloco diagonal de dimensōes $(m q \times m q)$.

Tomando-se o logaritmo de $L(\boldsymbol{\beta}, \mathbf{b} \mid \mathbf{y}, B, \Sigma)$, derivando-se e igualandose o resultado a zero, obtém-se o seguinte sistema

$$
\left[\begin{array}{cc}
\mathrm{X}^{\prime} \Sigma^{-1} \mathrm{X} & \mathrm{X}^{\prime} \Sigma^{-1} \mathrm{Z} \\
\mathrm{Z}^{\prime} \Sigma^{-1} \mathrm{X} & \mathrm{Z}^{\prime} \Sigma^{-1} \mathrm{Z}+\mathrm{B}^{-1}
\end{array}\right]\left[\begin{array}{c}
\beta^{0} \\
\hat{\mathrm{b}}
\end{array}\right]=\left[\begin{array}{c}
\mathrm{X}^{\prime} \Sigma^{-1} \mathrm{y} \\
\mathrm{Z}^{\prime} \Sigma^{-1} \mathrm{y}
\end{array}\right],
$$

que é denominado de Equações de Modelos Mistos, a partir das quais podem ser obtidas as soluções

$$
\boldsymbol{\beta}^{0}=\left\{\mathrm{X}^{\prime} V^{-1} \mathrm{X}\right\}^{-} \mathrm{X}^{\prime} V^{-1} \mathrm{y}
$$

onde $V^{-1}=\Sigma^{-1}-\Sigma^{-1} \mathrm{Z}\left(\mathrm{Z}^{\prime} \Sigma^{-1} \mathrm{Z}+\mathrm{B}^{-1}\right)^{-1} \mathrm{Z}^{\prime} \Sigma^{-1}$, e

$$
\hat{\mathrm{b}}=\left(\mathrm{Z}^{\prime} \Sigma^{-1} \mathrm{Z}+\mathrm{B}^{-1}\right)^{-1} \mathrm{Z}^{\prime} \Sigma^{-1}\left(\mathrm{y}-\mathrm{X} \beta^{0}\right) .
$$

Pode-se mostrar (Henderson, 1984) que a solução $\beta^{0}$ obtida anteriormente é também uma solução de mínimos quadrados generalizados (GLS) para o 
modelo

$$
\mathrm{y}=\mathrm{X} \boldsymbol{\beta}+\epsilon,
$$

onde $\boldsymbol{\epsilon}=\mathbf{Z} \mathbf{b}+\mathbf{e}, \boldsymbol{\epsilon} \sim N(\mathbf{0}, \mathrm{V})$, com $\mathrm{V}=\mathrm{Z}^{\prime} \mathrm{BZ}+\mathbf{\Sigma}$.

Uma interessante propriedade das soluções anteriormente apresentadas é que $\hat{\mathbf{b}}$ é o Melhor Preditor Linear Não-Viesado (BLUP) de b, e $L^{\prime} \boldsymbol{\beta}^{0}$ é o Melhor Estimador Linear Não-Viesado (BLUE) de uma função estimável $L^{\prime} \boldsymbol{\beta}$.

\subsubsection{Estimação dos componentes de variância}

Como verificado anteriormente, as soluções das Equações de Modelos Mistos requerem o conhecimento dos componentes de variância, os quais usualmente não são conhecidos. Não existe uma solução satisfatória para esse problema dentro de um ponto de vista frequentista. Uma prática comum para contornar essa situação é a estimação dos componentes de variância e a utilização de suas estimativas como os verdadeiros valores paramétricos na obtenção dos BLUE e BLUP. As propriedades dos estimadores e preditores resultantes a partir deste procedimento em dois estágios não são bem definidas, à excessão da propriedade de serem não-viesados (Kackar \& Harville, 1981).

Diversas metodologias foram propostas para a estimação dos componentes de variância em modelos lineares mistos. Uma abrangente discussão acerca destes métodos pode ser obtida em Searle et al. (1992).

Um importante trabalho com relação à estimação de componentes de variância é o de Henderson (1953), no qual são apresentados três métodos (Métodos I, II e III de Henderson) para diferentes modelos. Todos esses três métodos são análogos ao método de análise de variância (ANOVA) em casos de dados balanceados, no qual são igualados os quadrados médios às suas respectivas esperanças. Uma propriedade importante dos estimadores propostos por Henderson é que são não-viesados.

Uma outra classe de estimadores de variâncias que se tornou popular engloba os estimadores quadráticos não-viesados de norma (variância) mínima, ou MINQUE (MIVQUE) (La Motte, 1970, 1971, 1973; Rao, 1971a, b). Os estimadores 
MINQUE requerem normalidade dos dados, enquanto os MIVQUE não, sendo que estes correspondem aos anteriores sob a suposição gaussiana.

Atualmente, os métodos de máxima verossimilhança têm sido os preferidos. O princípio geral de Máxima Verossimilhança (ML) de Fisher, estendido por Hartley \& Rao (1967) para estimação de componentes de variância em modelos lineares mistos, é discutido por Harville (1977). Thompson $(1962,1963)$ e Patterson \& Thompson (1971) consideram o problema de maximização da parte da função de verossimilhança que é independente dos efeitos fixos. Este método considera os graus de liberdade utilizados na estimação dos efeitos fixos e é conhecido por Método de Máxima Verossimilhança Restrita (REML).

\subsection{Inferência bayesiana}

A inferência bayesiana sobre determinado vetor de parâmetros $\theta$ baseia-se em proposições probabilísticas condicionais a valores observados $\mathrm{y}$, escritas como a distribuição (ou densidade) a posteriori de $\boldsymbol{\theta}$, denotada por $p(\boldsymbol{\theta} \mid \mathbf{y})$.

Para se obter a distribuição a posteriori de $\boldsymbol{\theta}, p(\boldsymbol{\theta} \mid \mathbf{y})$, há a necessidade de iniciar-se com um modelo que forneça a distribuição de probabilidade conjunta de $\theta$ e y, a qual pode ser escrita como um produto de duas densidades

$$
p(\boldsymbol{\theta}, \mathbf{y})=p(\boldsymbol{\theta}) p(\mathbf{y} \mid \boldsymbol{\theta})
$$

as quais são denominadas distribuição a priori, $p(\boldsymbol{\theta})$, e distribuição amostral, $p(\mathbf{y} \mid \boldsymbol{\theta})$.

Simplesmente condicionando-se ao valor conhecido de $\mathbf{y}$, utilizando-se a propriedade básica de probabilidades condicionais, conhecida por Regra de Bayes, tem-se a densidade a posteriori

$$
p(\boldsymbol{\theta} \mid \mathbf{y})=\frac{p(\boldsymbol{\theta}, \mathbf{y})}{p(\mathbf{y})}=\frac{p(\boldsymbol{\theta}) p(\mathbf{y} \mid \boldsymbol{\theta})}{p(\mathbf{y})}
$$

onde $p(\mathrm{y})=\sum_{\theta} p(\theta) p(\mathbf{y} \mid \theta)$, com o somatório abrangendo todos os valores possíveis de $\boldsymbol{\theta}$ (ou $p(\mathbf{y})=\int_{\theta} p(\boldsymbol{\theta}) p(\mathbf{y} \mid \boldsymbol{\theta}) d \boldsymbol{\theta}$ para o caso de $\boldsymbol{\theta}$ contínuo).

Uma forma equivalente da expressão anterior omite o fator $p(\mathbf{y})$, o qual não depende de $\theta$ e, com y fixo, pode ser considerado constante, fornecendo a 
denominada densidade a posteriori não-normalizada, que é dada pela parte da direita da expressão

$$
p(\boldsymbol{\theta} \mid \mathbf{y}) \propto p(\boldsymbol{\theta}) p(\mathbf{y} \mid \boldsymbol{\theta}) .
$$

Tem-se, então, que a Regra de Bayes pode ser utilizada para combinar a informação contida nos dados com a probabilidade a priori.

Dentro da filosofia bayesiana, não há distinção entre estimação de efeitos fixos, predição de efeitos aleatórios, ou estimação de componentes de variância, pois todo e qualquer parâmetro do modelo é tratado como uma variável aleatória.

Dessa maneira, trabalhando-se dentro deste contexto com modelos lineares mistos, tem-se $\boldsymbol{\theta}$ como um vetor de quantidades não-observáveis, englobando tanto efeitos fixos quanto aleatórios, bem como os componentes de variância.

Para se inferir com relação a qualquer elemento de $\theta$, a distribuição $a$ posteriori conjunta dos parâmetros, $p(\theta \mid \mathrm{y})$, deve ser integrada em relação a todos os outros elementos que a constituem. Assim, se o interesse do pesquisador se concentra sobre determinado subconjunto de $\boldsymbol{\theta}, \boldsymbol{\theta}_{1}$, tem-se a necessidade da obtenção da distribuição $p\left(\boldsymbol{\theta}_{1} \mid \mathbf{y}\right)$, dada por

$$
p\left(\boldsymbol{\theta}_{1} \mid \mathbf{y}\right)=\int_{\theta \neq \theta_{1}} p(\boldsymbol{\theta} \mid \mathbf{y}) d \boldsymbol{\theta}_{\theta \neq \theta_{1}} .
$$

Essa operação, para obtenção da chamada distribuição a posteriori marginal do parâmetro (ou vetor) de interesse é, em geral, impossível de ser efetuada analiticamente com modelos mais complexos.

Algumas soluções para esse tipo de problema são sugeridas como, por exemplo, a aproximação normal (ou mesmo utilizando a distribuição $t$ de Student) ao redor da moda de $p(\boldsymbol{\theta} \mid \mathbf{y})$ (Tanner, 1993), ou a utilização de métodos de Monte Carlo, mais especificamente Cadeias de Markov, para a simulação de valores de distribuição estacionária que converge para $p(\theta \mid \mathrm{y})$ (Gelfand \& Smith, 1990; Gilks et al., 1996).

Dentre os métodos de Monte Carlo via Cadeias de Markov (MCMC), aqueles derivados do Algoritmo de Metropolis-Hastings, como por exemplo o Amostrador de Gibbs, têm se mostrado bastante úteis e eficientes em vários problemas multidimensionais (Gelfand et al., 1990). 


\subsection{Amostrador de Gibbs}

O Amostrador de Gibbs (Geman \& Geman, 1984) é um método de amostragem condicional alternada definido em termos de subvetores de $\theta$.

Suponha que o vetor $\theta$ possa ser dividido em $r$ componentes ou subvetores, ou seja, $\boldsymbol{\theta}=\left(\boldsymbol{\theta}_{1}^{\prime}, \boldsymbol{\theta}_{2}^{\prime}, \ldots, \boldsymbol{\theta}_{r}^{\prime}\right)^{\prime}$. Cada iteração do Amostrador de Gibbs consiste em simular valores de cada subvetor de $\boldsymbol{\theta}$, condicionalmente aos valores de todos os outros subvetores, totalizando $k$ simulações para cada iteração completa do ciclo.

Assim, para dado valor inicial arbitrário $\theta^{(0)}=\left(\theta_{1}^{(0)^{\prime}}, \theta_{2}^{(0)^{\prime}}, \ldots, \theta_{r}^{(0)^{\prime}}\right)^{\prime}$, inicia-se a primeira iteração do Amostrador de Gibbs simulando-se um novo valor para $\theta_{1}, \theta_{1}^{(1)}$, condicionalmente aos valores $\theta_{2}^{(0)}, \theta_{3}^{(0)}, \ldots, \theta_{r}^{(0)}$, seguindo-se de um novo valor para $\theta_{2}, \theta_{2}^{(1)}$, condicionalmente a $\theta_{1}^{(1)}, \theta_{3}^{(0)}, \ldots, \theta_{r}^{(0)}$, e assim por diante até a simulação de $\theta_{r}^{(1)}$, condicionalmente aos valores de $\theta_{1}^{(1)}, \theta_{2}^{(1)}, \ldots, \theta_{r-1}^{(1)}$, para se completar a primeira iteração do processo. A partir deste novo valor de $\theta$ simulado, inicia-se uma segunda iteração e assim por diante.

Pode-se demonstrar que, sob condições normais (Geman \& Geman, 1984), após um grande número de iterações, a sequência de valores gerados pelo Amostrador de Gibbs converge para uma distribuição estacionária igual a $p(\theta \mid \mathbf{y})$, ou seja, cada valor de $\theta$ obtido pelo Amostrador de Gibbs após convergência, é um valor simulado da distribuição conjunta de seus elementos.

Suponha que se tenha interesse na distribuição marginal de determinada função dos parâmetros $\phi=g(\boldsymbol{\theta})=g\left(\boldsymbol{\theta}_{1}, \boldsymbol{\theta}_{2}, \ldots, \boldsymbol{\theta}_{r}\right)$. Após obtida a convergência do Amostrador de Gibbs, a avaliação de $g($.$) para cada valor de \theta$ simulado fornece amostras de $\phi$, as quais podem ser utilizadas para estimação de densidade, utilizandose por exemplo algum método de estimação por meio de núcleo (kernel).

Esse tipo de aproximação, evidentemente, será tanto melhor quanto maior for o número de amostras utilizadas nos cálculos. Ainda, por se tratar de um processo de Markov, amostras sucessivas são dependentes entre si, e por essa razão a utilização de amostras obtidas a partir do descarte de algumas iterações intermediárias, pode também ser recomendada no sentido da obtenção de amostras 
independentes.

Assim, num processo de Amostragem Gibbs, o número total de iterações a partir de valor inicial $\theta^{(0)}$ é determinado a partir do número de iterações necessárias para convergẽncia, do número de iterações descartadas entre cada amostra utilizada nos cálculos e do número total de amostras que se deseja para as aproximações de Monte Carlo.

Uma abrangente apresentação acerca de critérios de convergência para sequências de Amostragem Gibbs pode ser obtida em Raftery \& Lewis (1992) e Best et al. (1995). Chib \& Carlin (1997) discutem estratégias para se acelerar esse processo, bem como para a obtenção de amostras Gibbs independentes em modelos lineares mistos.

\subsection{Distribuições normal/independentes}

\subsubsection{Definição}

As distribuições normal/independentes oferecem um interessante grupo de distribuições de caudas longas (leptocúrticas) que são comumente utilizadas para estimação robusta (Rogers \& Tukey, 1972; Andrews \& Mallows, 1974). Elas recebem este nome por serem derivadas a partir de uma relação entre uma distribuição normal e uma outra distribuição independente, referente a uma variável estritamente positiva.

Uma distribuição normal/independente (Dempster et al., 1980; Lange \& Sinsheimer, 1993) pode ser definida como a distribuição de um vetor aleatório $k$-dimensional $\mathrm{y}=\boldsymbol{\mu}+\mathrm{e} / \sqrt{w}$, onde $\boldsymbol{\mu}$ é o vetor de locação, de dimensão $k, w$ é uma variável aleatória positiva escalar com densidade $p(w \mid \nu)$, onde $\nu$ é um hiperparâmetro, e e é um vetor aleatório de dimensão $k$, normalmente distribuído com média 0 e matriz não-singular de covariâncias $\Sigma$. Sob esta situação, condicionalmente à $w$ y é normalmente distribuído com média $\mu$ e matriz de covariâncias $\Sigma / w$. 
A distribuição marginal de y é dada por

$$
p_{N / I}(\mathbf{y} \mid \boldsymbol{\mu}, \Sigma, \nu)=\int p_{N}(\mathbf{y} \mid \boldsymbol{\mu}, \Sigma, w) p_{I}(w \mid \nu) d w
$$

onde $p_{N}(),. p_{I}($.$) e p_{N / I}($.$) denotam densidades de probabilidade relativas à dis-$ tribuição normal, distribuição de $w$ (distribuição independente) e distribuição normal/independente, respectivamente.

A distribuição $p_{N / I}(\mathbf{y} \mid \boldsymbol{\mu}, \Sigma, \nu)$ tem as seguintes características (Lange \& Sinsheimer, 1993):

1. $E[\mathbf{y} \mid \boldsymbol{\mu}, \Sigma, \nu]=\boldsymbol{\mu}$, se ela existir; ·

2. $\operatorname{Var}[\mathbf{y} \mid \boldsymbol{\mu}, \Sigma, \nu]=E\left[w^{-1} \mid \nu\right] \Sigma$, se ela existir;

3. $p_{N / I}(\mathbf{y} \mid \boldsymbol{\mu}, \Sigma, \nu)$ é simétrica e unimodal, de maneira que a moda e a mediana também são iguais a $\mu$.

A seguir são discutidas algumas das propriedades das distribuições normal/independentes e, posteriormente, são apresentados alguns exemplos de casos específicos mais comuns, como a distribuição $t$ de Student, a distribuição slash e a distribuição normal contaminada.

\subsubsection{Algumas propriedades}

Sob as suposições anteriormente descritas com relação à definição de uma distribuição normal/independente, têm-se algumas propriedades interessantes, muitas delas idênticas àquelas asseguradas para a distribuição normal multivariada, como apresentado por Zellner (1976) para o caso da distribuição $t$.

Teorema 1. Seja $\mathbf{y} \sim p_{N / I}(\mathbf{y} \mid \boldsymbol{\mu}, \Sigma, \nu)$ um vetor aleatório normal/independente e $\mathbf{y}=\left[\mathbf{y}_{1}^{\prime}, \mathbf{y}_{2}^{\prime}\right]^{\prime}$ qualquer partição de $\mathbf{y}$ em dois componentes tendo $k_{1}$ e $k_{2}$ 
elementos, respectivamente. Os parâmetros $\boldsymbol{\mu}$ e $\Sigma$ também podem ser particionados correspondentemente. A distribuição marginal de $\mathbf{y}_{1} \sim p\left(\mathbf{y}_{1} \mid \boldsymbol{\mu}_{1}, \Sigma_{11}, \nu\right)$ também será normal/independente da mesma família da distribuição de y.

Prova: A distribuição marginal de $\mathrm{y}_{1}$ pode ser obtida a partir do modelo (2) de mistura de distribuições normais para $\mathbf{y}$, integrando-se em relação a $\mathrm{y}_{2}$, ou seja

$$
\begin{aligned}
p\left(\mathbf{y}_{1} \mid \boldsymbol{\mu}_{1}, \Sigma_{11}, \nu\right) & =\int p_{N / I}(\mathbf{y} \mid \boldsymbol{\mu}, \Sigma, \nu) d \mathbf{y}_{2} \\
& =\iint p_{N}(\mathbf{y} \mid \boldsymbol{\mu}, \Sigma, w) p_{I}(w \mid \nu) d w d \mathbf{y}_{2} \\
& =\int p_{I}(w \mid \nu)\left[\int p_{N}(\mathbf{y} \mid \boldsymbol{\mu}, \Sigma, w) d \mathbf{y}_{2}\right] d w .
\end{aligned}
$$

A distribuição $p_{N}(\mathbf{y} \mid \boldsymbol{\mu}, \Sigma, w)$, como definida anteriormente, referese à densidade de uma distribuição normal multivariada com média $\boldsymbol{\mu}$ e matriz de covariâncias $w^{-1} \Sigma \mathrm{e}$, portanto, a distribuição marginal de qualquer subvetor de $\mathrm{y}$ também terá distribuição normal. Assim,

$$
p\left(\mathbf{y}_{1} \mid \boldsymbol{\mu}_{1}, \Sigma_{11}, \nu\right)=\int p_{I}(w \mid \nu) p_{N}\left(\mathbf{y}_{1} \mid \boldsymbol{\mu}_{1}, \Sigma_{11}, w\right) d w,
$$

o que define uma distribuição normal/independente, da mesma família daquela relativa a y, uma vez que a distribuição independente de $w \mid \nu$ é a mesma para ambas.

Teorema 2. Seja $\mathbf{y} \sim p_{N / I}(\mathbf{y} \mid \boldsymbol{\mu}, \Sigma, \nu)$ um vetor aleatório normal/independente. Qualquer combinação linear de y tem distribuição normal/independente da mesma família da distribuição de $\mathbf{y}$.

Prova: Seja $\mathrm{x}=A y$ uma combinação linear de $\mathrm{y}$, onde $A$ é uma matriz não-singular de constantes, de dimensões $(k \times k)$. A densidade de $\mathbf{x}=A \mathbf{y}$, $g(\mathbf{x} \mid \boldsymbol{\mu}, \Sigma, \nu), \mathrm{e}$

$$
g(\mathbf{x} \mid \boldsymbol{\mu}, \Sigma, \nu)=p_{N / I}\left(A^{-1} \mathbf{x} \mid \mu, \Sigma, \nu\right)\left|A^{-1}\right|
$$




$$
\begin{aligned}
& =\int p_{N}\left(A^{-1} \mathbf{x} \mid \boldsymbol{\mu}, \Sigma, w\right) p_{I}(w \mid \nu)\left|A^{-1}\right| d w \\
& =\int(2 \pi)^{k / 2} w^{k / 2}|\Sigma|^{-1 / 2} \\
& \times \exp \left\{-\frac{w}{2}\left(A^{-1} \mathbf{x}-\boldsymbol{\mu}\right)^{\prime} \Sigma^{-1}\left(A^{-1} \mathbf{x}-\boldsymbol{\mu}\right)\right\} p_{I}(w \mid \nu)\left|A^{-1}\right| d w \\
& =\int(2 \pi)^{k / 2} w^{k / 2}|\Sigma|^{-1 / 2}\left|A^{-1}\right| \\
& \times \exp \left\{-\frac{w}{2}(\mathbf{x}-A \boldsymbol{\mu})^{\prime} A^{\prime}-1 \Sigma^{-1} A^{-1}(\mathbf{x}-A \boldsymbol{\mu})\right\} p_{I}(w \mid \nu) d w \\
& =\int(2 \pi)^{k / 2} w^{k / 2}\left|A \Sigma A^{\prime}\right|^{-1 / 2} \\
& \times \exp \left\{-\frac{w}{2}(\mathbf{x}-A \boldsymbol{\mu})^{\prime}\left(A \Sigma^{-1} A^{\prime}\right)^{-1}(\mathbf{x}-A \boldsymbol{\mu})\right\} p_{I}(w \mid \nu) d w \\
& =\int p_{N}\left(\mathbf{x} \mid A \boldsymbol{\mu}, A \Sigma A^{\prime}, w\right) p_{I}(w \mid \nu) d w \\
& =p_{N / I}\left(\mathbf{x} \mid A \boldsymbol{\mu}, A \Sigma A^{\prime}, \nu\right)
\end{aligned}
$$

caracterizando assim uma distribuição normal/independente da mesma família da distribuição de y, com média $A \boldsymbol{\mu}$, parâmetro de escala $A \Sigma A^{\prime}$ e hiperparâmetro $\nu$.

Analogamente, se $\mathrm{x}=\mathbf{a}^{\prime} \mathrm{y}$, onde $\mathbf{a}$ é um vetor coluna de constantes de dimensão $k$, tem-se que a distribuição de $\mathbf{x}$ é normal/independente univariada, da mesma família da distribuição de $\mathbf{y}$, com média a' $\boldsymbol{\mu}$, parâmetro de escala a' $\Sigma$ a e hiperparâmetro $\nu$.

Teorema 3. Seja y $\sim p_{N / I}(\mathrm{y} \mid \boldsymbol{\mu}, \Sigma, \nu)$ um vetor aleatório normal/independente, com $\Sigma=D$, uma matriz diagonal com elementos positivos. Quaisquer dois elementos de $\mathbf{y}, y_{1}$ e $y_{2}$, são não correlacionados, mas não independentes.

Prova: Duas variáveis aleatórias são não correlacionadas se a covariância entre elas for igual a zero.

Sabe-se que a matriz de covariâncias de $\mathrm{y}$, como visto anteriormente, é igual a $E\left[w^{-1} \mid \nu\right] \Sigma$ e, portanto, se $\Sigma=D, \operatorname{Var}[\mathrm{y} \mid \boldsymbol{\mu}, \Sigma, \nu]$ é também expressa por uma matriz diagonal $E\left[w^{-1} \mid \nu\right] D$, o que caracteriza y como um vetor de variáveis não correlacionadas. 
Duas variáveis aleatórias $y_{1}$ e $y_{2}$ são independentes se, e somente se, $p\left(y_{1}, y_{2}\right)=p\left(y_{1}\right) p\left(y_{2}\right)$, ou seja, a densidade conjunta das mesmas pode ser expressa como o produto das marginais correspondentes.

Assim, para o caso de dois elementos não correlacionados de $\mathbf{y}, y_{1} \mathrm{e}$ $y_{2}$, a independência implica que

$$
p_{N / I}\left(y_{1}, y_{2} \mid \boldsymbol{\mu}, D, \nu\right)=p_{N / I}\left(y_{1} \mid \mu_{1}, d_{1}, \nu\right) p_{N / I}\left(y_{2} \mid \mu_{2}, d_{2}, \nu\right) .
$$

É suficiente mostrar que a expressão acima não é verdadeira para valores particulares de $y_{1}$ e $y_{2}$, para comprovar a dependência entre as mesmas.

Sejam $y_{1}=\mu_{1}$ e $y_{2}=\mu_{2}$, ou seja, as variáveis assumindo os valores de suas esperanças. O produto das densidades marginais avaliado nas médias $\mu_{1}$ e $\mu_{2}$ é

$$
\begin{aligned}
\left.\left.p_{N / I}\left(y_{1} \mid \mu_{1}, d_{1}, \nu\right)\right|_{y_{1}=\mu_{1}} p_{N / I}\left(y_{2} \mid \mu_{2}, d_{2}, \nu\right)\right|_{y_{2}=\mu_{2}} \\
=\left.\left.\int p_{N}\left(y_{1} \mid \mu_{1}, d_{1}, w\right)\right|_{y_{1}=\mu_{1}} p_{I}(w \mid \nu) d w \int p_{N}\left(y_{2} \mid \mu_{2}, d_{2}, w\right)\right|_{y_{2}=\mu_{2}} p_{I}(w \mid \nu) d w \\
=\int\left(2 \pi d_{1}\right)^{-1 / 2} w^{1 / 2} p_{I}(w \mid \nu) d w \int\left(2 \pi d_{2}\right)^{-1 / 2} w^{1 / 2} p_{I}(w \mid \nu) d w \\
=(2 \pi)^{-1}\left(d_{1} d_{2}\right)^{-1 / 2}\left\{\int w^{1 / 2} p_{I}(w \mid \nu) d w\right\}^{2} \\
=(2 \pi)^{-1}\left(d_{1} d_{2}\right)^{-1 / 2}\left\{E\left[w^{1 / 2} \mid \nu\right]\right\}^{2},
\end{aligned}
$$

enquanto a distribuição conjunta avaliada nas médias é

$$
\begin{aligned}
\left.p_{N / I}\left(y_{1}, y_{2} \mid \boldsymbol{\mu}, D, \nu\right)\right|_{y_{1}=\mu_{1}, y_{2}=\mu_{2}} & =\left.\int p_{N}\left(y_{1}, y_{2} \mid \boldsymbol{\mu}, D, \nu\right)\right|_{y_{1}=\mu_{1}, y_{2}=\mu_{2}} p_{I}(w \mid \nu) d w \\
& =\int(2 \pi)^{-1} w\left(d_{1} d_{2}\right)^{-1 / 2} p_{I}(w \mid \nu) d w \\
& =(2 \pi)^{-1}\left(d_{1} d_{2}\right)^{-1 / 2} E[w \mid \nu] .
\end{aligned}
$$

As expressões (3) e (4) somente são iguais em casos muitos especiais para valores extremos de $\nu$, nos quais as distribuições normal/independentes coincidem com a distribuição normal, para os quais $w$ é uma constante, ou ainda em situações patológicas como, por exemplo, se $d_{1}=d_{2}=0$. 
Desta maneira, sob estas condições, embora $y_{1}$ e $y_{2}$ sejam não correlacionadas, estas variáveis não são independentes.

A seguir são apresentados alguns exemplos de distribuições normal/independentes mais usuais.

\subsubsection{Distribuição $t$ de Student}

A distribuição multivariada $t$ de Student $t_{k}(\boldsymbol{\mu}, \Sigma, \nu)$, onde $\nu$ é o número de graus de liberdade, pode ser derivada do modelo de mistura dado em (2), com $w$ distribuída como $\operatorname{Gama}(\nu / 2, \nu / 2)$, ou seja

$$
p(w \mid \nu)=\frac{(\nu / 2)^{\nu / 2}}{\Gamma(\nu / 2)} w^{\nu / 2-1} \exp \left\{-\frac{\nu}{2} w\right\}
$$

onde $\nu>0, w>0$, e $\Gamma($.) é a função gama padrão.

A distribuição $t$ de Student é talvez a mais conhecida e utilizada dentro do contexto de estimação robusta, dada a sua simplicidade e o fato de sua robustez ser definida por somente um único parâmetro, graus de liberdade (Lange et al., 1989). A distribuição normal é um caso particular da distribuição $t$ quando $\nu \rightarrow \infty$.

Um dos trabalhos que propiciaram o desenvolvimento teórico e sugerem exemplos de aplicações da distribuição $t$, dentro de um contexto frequentista, é o de Dempster et al. (1977), o qual mostra a utilização do Algoritmo Esperança-Maximização (EM) para a obtenção de estimativas de máxima verossimilhança com dados univariados e valor fixo para os graus de liberdade, e foi posteriormente estendido para modelos de regressão por Dempster et al. (1980).

A generalização desses resultados para o caso multivariado foi apresentada por Rubin (1983) e Sutradhar \& Ali (1986), enquanto Little \& Rubin (1987) e Little (1988) apresentam o Algoritmo EM para situações com valores perdidos. Um caso ainda mais geral, no qual o número de graus de liberdade é considerado desconhecido, e portanto com interesse em sua estimação, é discutido por Lange et al. (1989), o qual apresenta estimativas de máxima verossimilhança conjunta para todos os parâmetros, também utilizando o Algoritmo EM. 
Mais recentemente, Liu \& Rubin (1995) e Liu (1997) discutem algumas extensões do Algoritmo EM, denominadas ECM e ECME, estudando a eficiência computacional e a taxa de convergência das mesmas.

Considerável progresso no desenvolvimento metodológico de estimação robusta utilizando-se a distribuição $t$ é também verificado dentro da perspectiva bayesiana. Um importante trabalho foi apresentado por Zellner (1976), discutindo análise bayesiana de modelos de regressão com distribuição $t$ multivariada para $o$ erro. Geweke (1993) considera a distribuição $t$ em modelos lineares utilizando o Amostrador de Gibbs na análise a posteriori e, utilizando exemplos de aplicações em séries temporais em macroeconomia, verifica a superioridade dos modelos $t \mathrm{em}$ relação aos gaussianos no ajuste dos dados.

Enfocando situações com valores perdidos em conjuntos de dados multivariados, Liu (1995) discorre sobre a utilização da distribuição $t$ como alternativa para inferência robusta, e apresenta interessante discussão com relação à distribuição a priori para os graus de liberdade.

Especificamente em modelos lineares mistos, já são encontrados alguns trabalhos com relação à utilização da distribuição $t$ como alternativa robusta à distribuição gaussiana.

Direcionando-se às aplicações em avaliação genética de animais, nas quais o interesse do pesquisador concentra-se principalmente na predição de efeitos aleatórios e estimação de funções dos componentes de variância, Strandén (1996) desenvolveu trabalho dentro de uma perspectiva bayesiana utilizando o Amostrador de Gibbs e aproximações laplacianas, em modelos uni e multivariados com número de graus de liberdade desconhecido. Analisando dados de bovinos leiteiros, Strandén \& Gianola (1997) mostram a superioridade da distribuição $t$ em relação à normal para os resíduos, tanto em modelos uni quanto bivariados, utilizando o Fator de Bayes.

Dentro de uma perspectiva frequentista, Pinheiro et al. (1998) discutem o Algoritmo EM para a estimação de máxima verossimilhança em modelos lineares mistos com distribuição $t$ para os resíduos e efeitos aleatórios na análise de dados longitudinais. 


\subsubsection{Distribuição slash}

Uma outra interessante distribuição normal/independente, a distribuição slash multivariada, surge quando a distribuição de $w$ dado $\nu$ tem função de densidade dada por (Lange \& Sinsheimer, 1993)

$$
p(w \mid \nu)=\nu w^{\nu-1}
$$

onde $0<w \leq 1$ e $\nu>0$.

A distribuição slash não é muito usual ou conhecida, apesar de ser uma alternativa para estimação robusta, tendo também a distribuição normal como um caso particular quando $\nu \rightarrow \infty$.

Liu (1996) utiliza a distribuição slash como um dos exemplos de distribuições normal/independentes em seu trabalho em análise de regressão multivariada robusta.

\subsubsection{Distribuição normal contaminada}

No caso da distribuição normal multivariada contaminada, $w$ é uma variável discreta concentrada em dois pontos. A distribuição de probabilidades de $w$, dado $\nu=(\lambda, \gamma)^{\prime}$, é

$$
p(w \mid \nu)=\left\{\begin{array}{ccc}
\gamma & , \text { se } & w=\lambda \\
1-\gamma & , \text { se } & w=1
\end{array}\right.
$$

com $0<\lambda<1$ e $0 \leq \gamma<1$.

É fácil notar que a distribuição normal é um caso particular da distribuição normal contaminada quando não existem elementos contaminantes, ou seja, $\gamma=0$.

Rubin (1983) apresenta discussão e aplicação da distribuição normal contaminada utilizando o Algoritmo EM para obtenção de estimativas de máxima verossimilhança para o vetor de médias e para a matriz de covariâncias para dados multivariados, o qual foi estendido por Little (1988) para situações com valores perdidos. 
Little (1988) apresenta exemplos de regressão multivariada em presença de valores discrepantes, nos quais compara os resultados de análise adotandose a distribuição normal com aqueles obtidos pela distribuição $t$, com quatro graus de liberdade, e pela distribuição normal contaminada como alternativas robustas.

Num contexto bayesiano, Liu (1996) discute também modelos de regressão robustos utilizando a distribuição normal contaminada, mas com uma extensão ainda mais interessante em relação aos trabalhos anteriores, pois considera situações onde os parâmetros $\gamma$ e $\lambda$ são também desconhecidos, e portanto de interesse em sua estimação.

\subsection{Modelos lineares mistos robustos}

$\mathrm{Na}$ maioria das aplicações de modelos lineares mistos é assumida a distribuição normal para os resíduos e para os efeitos aleatórios, o que os tornam sensíveis à presença de valores discrepantes nas observações (Rogers \& Tukey, 1972).

Essa suposição foi estudada por Verbeke \& Lesaffre (1996), utilizando dados simulados a partir de mistura de distribuições normais. Os autores verificaram que, se a suposição de normalidade for assumida sob essas circunstâncias, os resultados podem ser bastante insatisfatórios, principalmente com relação à predição de efeitos aleatórios.

Alguns trabalhos com modelos lineares mistos robustos utilizando a distribuição $t$ foram apresentados por Strandén (1996), numa perspetiva bayesiana, e por Pinheiro et al. (1998), com estimação de máxima verossimilhança. No primeiro trabalho, a distribuição $t$ é assumida tanto para os resíduos quanto para os efeitos aleatórios. Toda a abordagem se dá dentro de um contexto bayesiano, com a utilização de Amostragem Gibbs, e a aplicação da metodologia é ilustrada utilizando-se dados de produção de bovinos leiteiros para a avaliação genética dos animais.

No trabalho de Pinheiro et al. (1998), um modelo similar é apresentado utilizando-se estimação de máxima verossimilhança, com a aplicação da metodologia em análise de dados em um estudo longitudinal envolvendo medidas ortodônticas 
em crianças. Nessa apresentação, entretando, uma forte e restritiva imposição é utilizada, no que se refere à suposição de que as distribuições $t$ dos efeitos aleatórios e dos resíduos para indivíduos de um mesmo grupo experimental apresentam o mesmo número de graus de liberdade. Não obstante, é ainda assumido um mesmo valor para as variáveis ponderadoras dos vetores de efeitos aleatórios e de resíduos de cada indivíduo.

Em ambos os trabalhos, entretanto, o modelo linear misto gaussiano caracteriza-se como um caso particular do modelo robusto, de maneira que este último apresenta ajuste consideravelmente superior nas situações estudadas, nas quais observou-se a presença de valores discrepantes. 


\section{METODOLOGIA}

Neste capítulo será discutida uma extensão do modelo linear misto (1), utilizando-se distribuições normal/independentes para os efeitos aleatórios e para os resíduos do mesmo. Todas as definições discutidas anteriormente permanecem as mesmas, exceto que o modelo agora é dado por

$$
\mathrm{y}_{i}=X_{i} \beta+Z_{i} \gamma_{i}+\varepsilon_{i}
$$

onde $\gamma_{i}=\mathrm{b}_{i} / \sqrt{u_{i}}$ e $\varepsilon_{i}=\mathrm{e}_{i} / \sqrt{w_{i}}$, com $u_{i}$ e $w_{i}$ sendo variáveis aleatórias independentes e positivas, com densidades $p\left(u_{i} \mid \lambda_{i}\right)$ e $p\left(w_{i} \mid \nu_{i}\right)$, respectivamente, e independentes de $\mathbf{b}_{i}$ e $\mathbf{e}_{i}$. Essas distribuições dependem dos parâmetros $\lambda_{i}$ e $\nu_{i}$, os quais podem ser tratados como conhecidos ou desconhecidos. Nesse último caso, algumas restrições são necessárias para torná-los estimáveis. Em geral, assume-se que $\lambda_{i}=\lambda_{h(i)}$ e $\nu_{i}=\nu_{h(i)}$, onde $h(i) \in 1, \ldots, l$ denota o grupo ao qual o $i$-ésimo indivíduo pertence (Pinheiro et al. , 1998). Uma situação simples, com constantes $\lambda_{i}$ e $\nu_{i}$ para todos os grupos, i.e., $\lambda_{i}=\lambda$ e $\nu_{i}=\nu$, será discutida aqui, apesar dessa restrição ser atenuada num dos exemplos apresentados no Capítulo 4.

Como discutido no ítem 2.4.1, as distribuições de $u_{i}$ e $w_{i}$ definem as distribuições de $\gamma_{i}$ e $\varepsilon_{i}$, assim como a distribuição marginal de $\mathrm{y}_{i}$. O modelo (1) é um caso particular do modelo com distribuições normal/independentes dado acima quando $p\left(u_{i}=1\right)=p\left(w_{i}=1\right)=1, i=1, \ldots, m$.

Será assumido, a partir de agora, um modelo no qual $\Sigma_{i}=\sigma^{2} I_{\left(n_{i}\right)}$, como comentado no ítem 2.1.1. Nesse caso, $\Sigma_{i}$ depende de $i$ somente em relação à sua dimensão.

Toda a metodologia estatística será abordada dentro de um contexto bayesiano, como em Liu (1996), mas utilizando-se o Amostrador de Gibbs na condução da análise a posteriori dos parâmetros.

Condicionalmente a $\mathbf{u}=\left(u_{1}, \ldots, u_{m}\right)^{\prime}$ e $\mathbf{w}=\left(w_{1}, \ldots, w_{m}\right)^{\prime}$, algumas derivações são comuns para qualquer uma das distribuições normal/independentes e, por esse motivo, nos próximos itens serão apresentados aspectos comuns do mo- 
delo geral (5), deixando para o ítem 3.4 a discussão de particularidades de casos específicos.

\subsection{Modelo amostral e função de verossimilhança}

Assumindo-se uma amostra aleatória de tamanho $m$ do modelo descrito em (5), a densidade condicional de todas as observações, dados os parâmetros, é

$$
\begin{aligned}
p\left(\mathbf{y} \mid \boldsymbol{\beta}, \boldsymbol{\gamma}, \sigma^{2}, \mathbf{w}\right) & \propto\left(\sigma^{2}\right)^{-n / 2} \prod_{i=1}^{m}\left[w_{i}^{n_{i} / 2} \exp \left\{-\frac{w_{i}}{2 \sigma^{2}} \boldsymbol{\delta}_{i}^{\prime} \boldsymbol{\delta}_{i}\right\}\right] \\
& =\left(\sigma^{2}\right)^{-n / 2} \prod_{i=1}^{m}\left[w_{i}^{n_{i} / 2}\right] \exp \left\{-\frac{1}{2 \sigma^{2}} \sum_{i=1}^{m} w_{i} \boldsymbol{\delta}_{i}^{\prime} \boldsymbol{\delta}_{i}\right\}
\end{aligned}
$$

onde $\mathrm{y}=\left(\mathrm{y}_{1}^{\prime}, \ldots, \mathrm{y}_{m}^{\prime}\right)^{\prime}, \boldsymbol{\gamma}=\left(\gamma_{1}^{\prime}, \ldots, \gamma_{m}^{\prime}\right)^{\prime}, \delta_{i}=\mathrm{y}_{i}-X_{i} \beta-Z_{i} \gamma_{i}$, e $n=\sum_{i=1}^{m} n_{i}$.

\subsection{Distribuições a priori e densidade conjunta a posteriori}

Para se completar a especificação do modelo num contexto bayesiano, é assumido que a distribuição conjunta a priori de todos os parâmetros e demais variáveis não-observáveis do modelo têm densidade

$$
\begin{aligned}
p\left(\boldsymbol{\beta}, \boldsymbol{\gamma}, B, \mathbf{u}, \lambda, \sigma^{2}, \mathbf{w}, \nu \mid \mathbf{h}\right) & =p(\boldsymbol{\beta}) p\left(B \mid \varphi_{1}, S\right) p\left(\sigma^{2} \mid \varphi_{2}, s^{2}\right) \\
& \times \prod_{i=1}^{m}\left[p\left(\gamma_{i} \mid B, u_{i}\right) p\left(u_{i} \mid \lambda\right) p\left(w_{i} \mid \nu\right)\right] p\left(\lambda \mid \xi_{1}\right) p\left(\nu \mid \xi_{2}\right)
\end{aligned}
$$

onde $\mathbf{h}=\left(\varphi_{1}, S, \varphi_{2}, s^{2}, \xi_{1}, \xi_{2}\right)^{\prime}$ representa os hiperparâmetros.

Para se garantir que a distribuição a posteriori seja própria, todas as distribuições a priori serão próprias (Hobert \& Casella, 1996). Em geral, com modelos lineares mistos, são assumidas distribuições a priori conjugadas as quais apresentam, além da conveniência matemática, bastante flexibilidade na modelagem de sua forma.

Para os efeitos fixos, será assumida uma distribuição a priori uniforme, i.e.,

$$
p(\boldsymbol{\beta}) \propto \text { constante }
$$


$\operatorname{com} \boldsymbol{\beta}_{\min }<\boldsymbol{\beta}<\boldsymbol{\beta}_{\max }$.

Cada um dos efeitos aleatórios, condicionalmente aos pesos $u$, apresenta distribuição normal multivariada independente com média 0 e matriz de covariâncias $B / u_{i}$, então

$$
p\left(\boldsymbol{\gamma}_{i} \mid B, u_{i}\right) \propto u_{i}^{q / 2}|B|^{-1 / 2} \exp \left\{-\frac{u_{i}}{2} \gamma_{i}^{\prime} B^{-1} \gamma_{i}\right\} .
$$

A distribuição a priori da matriz de covariâncias B é proporcional a uma distribuição Wishart invertida $\operatorname{Inv}-$ Wishart $_{\varphi_{1}}\left(S^{-1}\right)$, i.e.,

$$
p\left(B \mid \varphi_{1}, S\right) \propto|B|^{-\left(\varphi_{1}+q+1\right) / 2} \exp \left\{-\frac{1}{2} \operatorname{tr}\left(S B^{-1}\right)\right\},
$$

onde $\operatorname{tr}($.$) é o traço, e B$ é positiva definida.

Para o componente de variância residual $\sigma^{2}$, uma distribuição Chiquadrado escalonada invertida, Inv $-\chi^{2}\left(\varphi_{2}, s^{2}\right)$, é adotada como sua distribuição $a$ priori, i.e.,

$$
p\left(\sigma^{2} \mid \varphi_{2}, s^{2}\right) \propto\left(\sigma^{2}\right)^{-\left(\varphi_{2}+2\right) / 2} \exp \left\{-\frac{1}{2} \frac{\varphi_{2} s^{2}}{\sigma^{2}}\right\} .
$$

As distribuições de $u_{i}$ e $w_{i}$, bem como as distribuições a priori de $\lambda$ e $\nu$, dependem da distribuição normal/independente assumida, e serão discutidas separadamente nos itens 3.4.1 a 3.4.3.

Toda inferência bayesiana é baseada na distribuição conjunta a posteriori dos parâmetros e demais variáveis não-observáveis, que é proporcional ao produto das densidades do modelo amostral e das distribuições a priori assumidas, ou seja

$$
p\left(\boldsymbol{\beta}, \boldsymbol{\gamma}, B, \mathbf{u}, \lambda, \sigma^{2}, \mathbf{w}, \nu \mid \mathbf{y}, \mathbf{h}\right) \propto p\left(\mathbf{y} \mid \boldsymbol{\beta}, \boldsymbol{\gamma}, \sigma^{2}, \mathbf{w}\right) p\left(\boldsymbol{\beta}, \boldsymbol{\gamma}, B, \mathbf{u}, \lambda, \sigma^{2}, \mathbf{w}, \nu \mid \mathbf{h}\right),
$$

a qual, pelas suposições descritas anteriormente, fica

$$
\begin{aligned}
p\left(\boldsymbol{\beta}, \boldsymbol{\gamma}, B, \mathbf{u}, \lambda, \sigma^{2}, \mathbf{w}, \nu \mid \mathbf{y}, \mathbf{h}\right) & \propto\left(\sigma^{2}\right)^{-\left(n+\varphi_{2}+2\right) / 2}|B|^{-\left(m+\varphi_{1}+q+1\right) / 2} \\
& \times \exp \left\{-\frac{1}{2 \sigma^{2}}\left(\varphi_{2} S^{2}+\sum_{i=1}^{m} w_{i} \delta_{i}^{\prime} \delta_{i}\right)\right\} \\
& \times \exp \left\{-\frac{1}{2}\left[\operatorname{tr}\left(S B^{-1}\right)+\sum_{i=1}^{m} u_{i} \gamma_{i}^{\prime} B^{-1} \gamma_{i}\right]\right\}
\end{aligned}
$$




$$
\begin{aligned}
& \times \prod_{i=1}^{m}\left[w_{i}^{n_{i} / 2} u_{i}^{q / 2} p\left(w_{i} \mid \nu\right) p\left(u_{i} \mid \lambda\right)\right] \\
& \times \quad p\left(\lambda \mid \xi_{1}\right) p\left(\nu \mid \xi_{2}\right) .
\end{aligned}
$$

A distribuição (6) é intratável analiticamente para a obtenção das inferências de interesse, de maneira que algum método numérico ou de Monte Carlo é necessário para se aproximar a análise a posteriori dos parâmetros. Neste trabalho será adotado o método de Amostragem Gibbs (Geman \& Geman, 1984; Gelfand \& Smith, 1990) para o qual são necessárias as distribuições condicionais completas a posteriori de cada um dos parâmetros ou blocos dos mesmos.

\subsection{Distribuições condicionais completas a posteriori}

A amostragem de Gibbs consiste de simulações alternadas de cada distribuição condicional dos parâmetros e variáveis não-observáveis de (6). Todas as distribuições condicionais relativas a valores fixos de $u$ e w são as mesmas para quaisquer das distribuições normal/independentes e também serão discutidas neste ítem. Aquelas distribuições condicionais relativas a casos específicos serão discutidas nos itens 3.4.1 a 3.4.3.

Para os parâmetros de locação, é adotada a estratégia de amostragem em blocos independentes, sendo um relativo ao vetor de efeitos fixos e os outros relativos aos vetores de efeitos aleatórios.

Para o vetor de efeitos fixos, a distribuição condicional completa a posteriori é uma distribuição normal multivariada com vetor de médias $\hat{\boldsymbol{\beta}}=\left(\sum_{i=1}^{m} w_{i} X_{i}^{\prime} X_{i}\right)^{-1}\left(\sum_{i=1}^{m} w_{i} X_{i}^{\prime}\left(\mathbf{y}_{i}-Z_{i} \boldsymbol{\gamma}_{i}\right)\right)$, e matriz de covariâncias $V_{\hat{\beta}}=\sigma^{2}\left(\sum_{i=1}^{m} w_{i} X_{i}^{\prime} X_{i}\right)^{-1}$.

No caso de cada vetor de efeitos aleatórios, a distribuição condicional completa a posteriori é também normal multivariada, com parâmetros $\hat{\gamma}_{i}=\left(Z_{i}^{\prime} Z_{i}+\frac{u_{i} \sigma^{2}}{w_{i}} B^{-1}\right)^{-1} Z_{i}^{\prime}\left(\mathrm{y}_{i}-X_{i} \beta\right)$ e $V_{\hat{\gamma}_{i}}=\left[\frac{w_{i}}{\sigma^{2}} Z_{i}^{\prime} Z_{i}+u_{i} B^{-1}\right]^{-1}$, a qual pode ser facilmente encontrada seguindo sugestão de pontos de dados adicionais (Gelman et al., 1995). 
Para os parâmetros de escala, tem-se que $B \mid \gamma, \mathrm{u}, \varphi_{1}, S$ tem distribuição Wishart invertida com parâmetros $\psi_{1}^{-1}=S+\sum_{i=1}^{m} u_{i} \gamma_{i} \gamma_{i}^{\prime}$ e $\lambda_{1}=m+\varphi_{1}$ (graus de liberdade), e $\sigma^{2} \mid \mathbf{y}, \boldsymbol{\beta}, \boldsymbol{\gamma}, \mathbf{w}, \varphi_{2}, s^{2}$ tem distribuição Chi-quadrado escalonada invertida com parâmetros $\psi_{2}=\frac{1}{\left(\varphi_{2}+n\right)}\left[\varphi_{2} s^{2}+\sum_{i=1}^{m} w_{i} \delta_{i}^{\prime} \delta_{i}\right]$ e $\lambda_{2}=n+\varphi_{2}$.

Para se completar a especificação necessária para a Amostragem Gibbs são necessárias as distribuições condicionais completas a posteriori

de cada uma das variáveis ponderadoras $u_{i}$ e $w_{i}$, e de seus respectivos hiperparâmetros $\lambda$ e $\nu$.

Para cada elemento de $u_{i}$, esta distribuição é

$$
p\left(u_{i} \mid \gamma_{i}, B, \lambda\right) \propto u_{i}^{q / 2} \exp \left\{-\frac{u_{i}}{2} \gamma_{i}^{\prime} B^{-1} \gamma_{i}\right\} p\left(u_{i} \mid \lambda\right)
$$

e para $w_{i}$, tem-se

$$
p\left(w_{i} \mid \mathbf{y}_{i}, \boldsymbol{\beta}, \gamma_{i}, \nu\right) \propto w_{i}^{n_{i} / 2} \exp \left\{-\frac{w_{i}}{2 \sigma^{2}} \delta_{i}^{\prime} \delta_{i}\right\} p\left(w_{i} \mid \nu\right)
$$

Para a distribuição condicional completa a posteriori de $\lambda$ e $\nu$, tem-se que

$$
p\left(\lambda \mid \mathbf{u}, \xi_{1}\right) \propto p\left(\lambda \mid \xi_{1}\right) \prod_{i=1}^{m} p\left(u_{i} \mid \lambda\right)
$$

e

$$
p\left(\nu \mid \mathbf{w}, \xi_{2}\right) \propto p\left(\nu \mid \xi_{2}\right) \prod_{i=1}^{m} p\left(w_{i} \mid \nu\right) .
$$

As expressões (7) a (10), como discutido anteriormente, somente podem ser completamente definidas pela escolha das distribuições normal/independentes de interesse, caracterizadas pelas distribuições de $\mathbf{u}$ dado $\lambda$ e $\mathbf{w}$ dado $\nu$, e as distribuições a priori de $\lambda$ e $\nu$.

\subsection{Exemplos com distribuições normal/independentes}

Neste ítem serão discutidas distribuiçōes a priori para $\lambda$ e $\nu$, bem como as distribuições condicionais completas a posteriori de $\mathbf{u}, \mathbf{w}, \lambda$ e $\nu$ para modelos referentes às distribuiçōes normal/independentes discutidas nos itens 2.4.3 a 2.4.5. 


\subsubsection{Distribuição $t$}

Alguns dos mais recentes trabalhos com distribuição $t$ em estimação robusta são Liu \& Rubin (1995), apresentando o Algoritmo EM e suas extensões, Geweke (1993), com modelos lineares numa perspectiva bayesiana, Liu (1995), tanto num contexto bayesiano como de máxima verossimilhança, e Welsh \& Richardson (1997), numa revisão de procedimentos de estimação robusta em modelos lineares.

Mais especificamente com modelos lineares mistos, a utilização da distribuição $t$ foi apresentada por Strandén (1996), num contexto bayesiano, e por Pinheiro et al. (1998), com estimação de máxima verossimilhança.

Como discutido no ítem 2.4.3, a distribuição $t$ pode ser derivada do modelo de mistura dado em (2), utilizando-se a distribuição gama para as variáveis ponderadoras. Algumas sugestões de distribuições a priori para os graus de liberdade (df) são discutidas em Liu (1995), e aqui será adotada uma distribuição uniforme para seu inverso, o que corresponde a

$$
p(d f) \propto d f^{-2}
$$

com $d f \geq 1$. Esse limite inferior para o domínio da distribuição dos graus de liberdade torna a mesma própria e corresponde à distribuição de Cauchy.

Neste sentido, quando $p\left(u_{i} \mid \lambda\right) \sim \operatorname{Gama}(\lambda / 2, \quad \lambda / 2)$, e $p(\lambda) \propto \lambda^{-2}$, as distribuições condicionais completas a posteriori de $u_{i}$ e $\lambda$ serão

$$
p\left(u_{i} \mid \gamma_{i}, B, \lambda\right) \propto \exp \left\{-\frac{u_{i}}{2}\left[\lambda+\gamma_{i}^{\prime} B^{-1} \gamma_{i}\right]\right\}
$$

o que corresponde a uma distribuição gama com parâmetros $(q+\lambda) / 2$ e $\frac{1}{2}\left[\lambda+\gamma_{i}^{\prime} B^{-1} \gamma_{i}\right], \mathrm{e}$

$$
\begin{aligned}
p(\lambda \mid \mathbf{u}) & \propto \frac{2^{-m \lambda / 2} \lambda^{m \lambda / 2-2}}{[\Gamma(\lambda / 2)]^{m}}\left(\prod_{i=1}^{m} u_{i}\right)^{\lambda / 2} \exp \left\{-\frac{\lambda}{2} \sum_{i=1}^{m} u_{i}\right\} \\
& =\pi(\lambda) \lambda^{m \lambda / 2-2} \exp \left\{-\frac{\lambda}{2} \sum_{i=1}^{m}\left(u_{i}-\log u_{i}\right)\right\}
\end{aligned}
$$

onde $\pi(\lambda)=\left[2^{\lambda / 2} \Gamma(\lambda / 2)\right]^{-m}$, i.e., $\lambda \mid \mathbf{u} \propto \pi(\lambda) \operatorname{Gama}\left(\frac{m \lambda}{2}-1, \quad \frac{1}{2} \sum_{i=1}^{m}\left(u_{i}-\log u_{i}\right)\right)$, respectivamente. 
A distribuição $p(\lambda \mid \mathrm{u})$ não apresenta uma forma fechada e, para se obter uma amostra da mesma num esquema de Amostragem Gibbs, pode ser utilizado o Algoritmo de Metropolis-Hastings (Hastings, 1970), ou mesmo Amostragem de Rejeição (Ripley, 1987), a qual mostra-se eficiente uma vez que $p(\lambda \mid \mathbf{u})$ é univariada (Geweke, 1993).

No caso da distribuição $t$ também para os vetores residuais, $p\left(w_{i} \mid \nu\right) \sim \operatorname{Gama}(\nu / 2, \nu / 2)$ e, utilizando-se novamente a distribuição a priori $p(\nu) \propto \nu^{-2}$, as distribuições condicionais completas a posteriori são

$$
p\left(w_{i} \mid \boldsymbol{\beta}, \boldsymbol{\gamma}, \sigma^{2}, \nu, \mathbf{y}\right) \propto w_{i}^{\left(n_{i}+\nu-2\right) / 2} \exp \left\{-\frac{w_{i}}{2}\left[\nu+\frac{1}{\sigma^{2}} \delta_{i}^{\prime} \delta_{i}\right]\right\}
$$

a qual corresponde a uma distribuição gama com parâmetros $\left(n_{i}+\nu\right) / 2$ e $\frac{1}{2}\left[\nu+\frac{1}{\sigma^{2}} \delta_{i}^{\prime} \delta_{i}\right], \mathrm{e}$

$$
\begin{aligned}
p(\nu \mid \mathbf{w}) & \propto \frac{2^{-m \nu / 2} \nu^{m \nu / 2-2}}{[\Gamma(\nu / 2)]^{m}}\left(\prod_{i=1}^{m} w_{i}\right)^{\nu / 2} \exp \left\{-\frac{\nu}{2} \sum_{i=1}^{m} w_{i}\right\} \\
& =\pi(\nu) \nu^{m \nu / 2-2} \exp \left\{-\frac{\nu}{2} \sum_{i=1}^{m}\left(w_{i}-\log w_{i}\right)\right\}
\end{aligned}
$$

onde $\pi(\nu)=\left[2^{\nu / 2} \Gamma(\nu / 2)\right]^{-m}$. Desta maneira, novamente $\nu / \mathrm{w}$ é proporcional a uma distribuição $\operatorname{Gama}\left(\frac{m \nu}{2}-1, \quad \frac{1}{2} \sum_{i=1}^{m}\left(w_{i}-\log w_{i}\right)\right)$, mas com uma função multiplicativa $\pi(\nu)$.

\subsubsection{Distribuiçāo slash}

Com distribuição slash (Rogers \& Tukey, 1972; Lange \& Sinsheimer, 1993) para os efeitos aleatórios, utilizando-se a distribuição $\operatorname{Gama}\left(a_{1}, b_{1}\right)$ como priori para $\lambda$, com valores positivos pequenos para $a_{1}$ e $b_{1}\left(b_{1}<<a_{1}\right)$, a qual é uma distribuição conjugada em relação a $u_{i}$, as distribuições condicionais completas $a$ posteriori de $u_{i}$ e $\lambda$ são

$$
p\left(u_{i} \mid \gamma_{i}, B, \lambda\right) \propto u_{i}^{q / 2+\lambda-1} \exp \left\{-\frac{u_{i}}{2} \gamma_{i}^{\prime} B^{-1} \gamma_{i}\right\}
$$


a qual corresponde a uma distribuição gama truncada, i.e.,

$$
u_{i} \mid \boldsymbol{\gamma}_{i}, B, \lambda \sim \underset{0<u_{i}<1}{\operatorname{Gama}-\operatorname{Truncada}}\left(\frac{q}{2}+\lambda, \quad \frac{1}{2} \gamma_{i}^{\prime} B^{-1} \boldsymbol{\gamma}_{i}\right)
$$

e

$$
\begin{aligned}
p\left(\lambda \mid \mathbf{u}, \boldsymbol{\xi}_{1}\right) & \propto \lambda^{m+a_{1}-1} \exp \left\{-b_{1} \lambda\right\} \prod_{i=1}^{m} u_{i}^{\lambda-1} \\
& =\lambda^{m+a_{1}-1} \exp \left\{-\lambda\left(b_{1}-\sum_{i=1}^{m} \log u_{i}\right)\right\}
\end{aligned}
$$

i.e., $\lambda \mid \mathbf{u}, \boldsymbol{\xi}_{1} \sim\left(m+a_{1}, \quad b_{1}-\sum_{i=1}^{m} \log u_{i}\right)$, onde $\boldsymbol{\xi}_{1}=\left(a_{1}, b_{1}\right)^{\prime}$, respectivamente.

Para resíduos slash, tem-se que $p\left(w_{i} \mid \nu\right)=\nu w_{i}^{\nu-1}$ e $\nu \mid \boldsymbol{\xi}_{2} \sim \operatorname{Gama}\left(a_{2}, b_{2}\right), \boldsymbol{\xi}_{2}=\left(a_{2}, b_{2}\right)^{\prime}$, obtendo-se as seguintes distribuições condicionais completas a posteriori

$$
p\left(w_{i} \mid \mathbf{y}_{i}, \boldsymbol{\beta}, \boldsymbol{\gamma}_{i}, \sigma^{2}, \nu\right) \propto w_{i}^{n_{i} / 2+\nu-1} \exp \left\{-\frac{w_{i}}{2 \sigma^{2}} \delta_{i}^{\prime} \delta_{i}\right\},
$$

correspondente a uma distribuição gama truncada com parâmetros $\frac{n_{i}}{2}+\nu$ e $-\frac{1}{2 \sigma^{2}} \delta_{i}^{\prime} \delta_{i}$, com $0<w_{i}<1$, e

$$
\begin{aligned}
p\left(\nu \mid \mathbf{w}, \boldsymbol{\xi}_{2}\right) & \propto \nu^{m+a_{2}-1} \exp \left\{-b_{2} \nu\right\} \prod_{i=1}^{m} w_{i}^{\nu-1} \\
& =\nu^{m+a_{2}-1} \exp \left\{-\nu\left(b_{2}-\sum_{i=1}^{m} \log w_{i}\right)\right\},
\end{aligned}
$$

i.e., $\nu \mid \mathbf{w}, \boldsymbol{\xi}_{2} \sim \operatorname{Gama}\left(m+a_{2}, b_{2}-\sum_{i=1}^{m} \log w_{i}\right)$.

\subsubsection{Distribuição normal contaminada}

A distribuição de probabilidades de $u_{i}$ no caso da distribuição normal contaminada para os efeitos aleatórios pode ser expressa como

$$
p\left(u_{i} \mid \nu_{1}\right)=\phi_{1}^{\left(\frac{1-u_{i}}{1-\tau_{1}}\right)}\left(1-\phi_{1}\right)^{\left(\frac{u_{i}-\tau_{1}}{1-\tau_{1}}\right)}
$$

$\operatorname{com} u_{i}=\left\{\tau_{1}, 1\right\}$ e $\nu_{1}=\left(\phi_{1}, \tau_{1}\right)^{\prime}$, onde $0<\tau_{1}<1$ e $0 \leq \phi_{1}<1$. 
Utilizando uma distribuição a priori Uniforme $(0,1)$ para $\tau_{1}$ e a conjugada $\operatorname{Beta}\left(a_{1}, b_{1}\right)$ como distribuição a priori para $\phi_{1}$, a distribuição condicional completa a posteriori para cada $u_{i}$ é

$$
p\left(u_{i} \mid \gamma_{i}, B, \lambda\right) \propto u_{i}^{q / 2} \phi_{1}^{\left(\frac{1-u_{i}}{1-\tau_{1}}\right)}\left(1-\phi_{1}\right)^{\left(\frac{u_{i}-\tau_{1}}{1-\tau_{1}}\right)} \exp \left\{-\frac{1}{2} u_{i} \gamma_{i}^{\prime} B^{-1} \gamma_{i}\right\},
$$

a qual pode ser escrita como sendo proporcional a

$$
\left\{\begin{array}{cl}
\tau_{1}^{q / 2} \phi_{1} \exp \left\{-\frac{\tau_{1}}{2} \gamma_{i}^{\prime} B^{-1} \gamma_{i}\right\} & , \text { se } u_{i}=\tau_{1} \\
\left(1-\phi_{1}\right) \exp \left\{-\frac{1}{2} \gamma_{i}^{\prime} B^{-1} \gamma_{i}\right\} & , \text { se } u_{i}=1
\end{array}\right.
$$

Para o hiperparâmetro $\phi_{1}$, a proporção de valores discrepantes (ou de elementos da população contaminante), sua distribuição condicional completa $a$ posteriorié

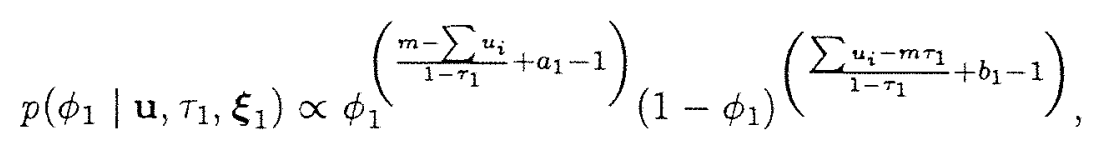

a qual corresponde a uma distribuição beta

$$
\phi_{1} \mid \mathbf{u}, \tau_{1}, \xi_{1} \sim B e t a\left[a_{1}+\frac{1}{1-\tau_{1}} \sum_{i=1}^{m}\left(1-u_{i}\right), \quad b_{1}+\frac{1}{1-\tau_{1}} \sum_{i=1}^{m}\left(u_{i}-\tau_{1}\right)\right],
$$

onde $\boldsymbol{\xi}_{1}=\left(a_{1}, b_{1}\right)^{\prime}$.

No caso do hiperparâmetro $\tau_{1}$, tem-se um problema de irredutibilidade, em decorrência de um confundimento entre esse parâmetro e $u$, no sentido de que, condicionalmente a $\mathbf{u}, \tau_{1}$ é completamente definido, assumindo o valor de qualquer $u_{i} \neq 1$, i.e., $\tau_{1}$ permanecerá tipicamente em seu valor inicial arbitrário utilizado no esquema de Amostragem Gibbs.

Para se acomodar essa situação, Liu (1996) sugere que u seja inicialmente integrado, e utiliza o Método de Rejeição para se obterem amostras da distribuição condicional de $\tau_{1}$.

Aqui será utilizado o Método de Metropolis-Hastings para se atualizar $\tau_{1}$ (M. Newton, 1998, comunicação pessoal), movendo de $\mathbf{S}_{1}=\left\{\tau_{1}, u_{1}, \ldots, u_{m}\right.$, outros $\}$ para $\mathbf{S}_{1}^{*}=\left\{\tau_{1}^{*}, u_{1}^{*}, \ldots, u_{m}^{*}\right.$ outros $\}$, onde

$$
\tau_{1}^{*} \sim B e t a[a, b] \text { e } u_{i}^{*}=\left\{\begin{array}{cc}
1, & \text { se } u_{i}=1 \\
\tau_{1}^{*}, & \text { se } u_{i}=\tau_{1}
\end{array},\right.
$$


com probabilidade dada pela relação de Hastings,

$$
r_{1}=\frac{\pi\left(\mathbf{S}_{1}^{*}\right) q\left(\mathbf{S}_{1}^{*}, \mathbf{S}_{1}\right)}{\pi\left(\mathbf{S}_{1}\right) q\left(\mathbf{S}_{1}, \mathbf{S}_{1}^{*}\right)}
$$

onde $\pi($.$) é a distribuição condicional completa a posteriori de \tau_{1}$ e $\mathbf{u}$,

$$
\begin{aligned}
\pi\left(\mathbf{S}_{1}\right) & =p\left(\tau_{1}, \mathbf{u} \mid \phi_{1}, B, \gamma\right) \\
& \propto\left[\prod_{i=1}^{m} u_{i}\right]^{q / 2} \phi_{1}^{\left(\frac{m-\sum u_{i}}{1-\tau_{1}}\right)}\left(1-\phi_{1}\right)^{\left(\frac{\sum u_{i}-m \tau_{1}}{1-\tau_{1}}\right)} \exp \left\{-\frac{1}{2} \sum_{i=1}^{m} u_{i} \gamma_{i}^{\prime} B^{-1} \gamma_{i}\right\}
\end{aligned}
$$

e $q($.$) , a densidade geradora de candidatos (proposal), assumida aqui como Beta[a,b].$

Assumindo-se também a distribuição normal contaminada para os resíduos e utilizando-se distribuiçōes a priori similares para $\nu_{2}=\left(\phi_{2}, \tau_{2}\right)^{\prime}$, i.e., $\tau_{2} \sim$ Uniforme $(0,1)$ e $\phi_{2} \mid \xi_{2} \sim \operatorname{Beta}\left(a_{2}, b_{2}\right)$, onde $\xi_{2}=\left(a_{2}, b_{2}\right)^{\prime}$, a distribuição condicional completa a posteriori para cada $w_{i}$ é

$$
\begin{aligned}
p\left(w_{i} \mid \mathbf{y}_{i}, \boldsymbol{\beta}, \gamma_{i}, \sigma^{2}, \nu_{2}\right) & \propto w_{i}^{n_{2} / 2} \phi_{2}^{\left(\frac{1-w_{i}}{1-\tau_{2}}\right)}\left(1-\phi_{2}\right)^{\left(\frac{w_{i}-\tau_{2}}{1-\tau_{2}}\right)} \\
& \times \exp \left\{-\frac{w_{i}}{2 \sigma^{2}} \delta_{i}^{\prime} \boldsymbol{\delta}_{i}\right\}
\end{aligned}
$$

a qual pode ser expressa como proporcional a

$$
\left\{\begin{array}{cc}
\tau_{2}^{n_{i} / 2} \phi_{2} \exp \left\{-\frac{\tau_{2}}{2 \sigma^{2}} \delta_{i}^{\prime} \delta_{i}\right\} & , \text { se } w_{i}=\tau_{2} \\
\left(1-\phi_{2}\right) \exp \left\{-\frac{1}{2 \sigma^{2}} \delta_{i}^{\prime} \delta_{i}\right\} & , \text { se } w_{i}=1
\end{array}\right.
$$

e, para o hiperparâmetro $\phi_{2}$, esta fica

$$
\phi_{2} \mid \mathrm{w}, \tau_{2}, \xi_{2} \sim \operatorname{Beta}\left[a_{2}+\frac{1}{1-\tau_{2}} \sum_{i=1}^{m}\left(1-w_{i}\right), \quad b_{2}+\frac{1}{1-\tau_{2}} \sum_{i=1}^{m}\left(w_{i}-\tau_{2}\right)\right] \text {. }
$$

O mesmo problema de irredutibilidade é encontrado para $\tau_{2}$, mas - mesmo esquema de Metropolis-Hastings pode ser utilizado também para essa situação. Neste caso, move-se de $\mathbf{S}_{2}=\left\{\tau_{2}, w_{1}, \ldots, w_{m}\right.$, outros $\}$ para $\mathbf{S}_{2}^{*}=\left\{\tau_{2}^{*}, w_{1}^{*}, \ldots, w_{m}^{*}\right.$, outros $\}$, onde

$$
r_{2}^{*} \sim \operatorname{Beta}[a, b] \text { e } w_{i}^{*}=\left\{\begin{array}{cc}
1, & \text { se } w_{i}=1 \\
\tau_{2}^{*}, & \text { se } w_{i}=\tau_{2}
\end{array},\right.
$$


com probabilidade dada por

$$
r_{2}=\frac{\pi\left(\mathbf{S}_{2}^{*}\right) q\left(\mathbf{S}_{2}^{*}, \mathbf{S}_{2}\right)}{\pi\left(\mathbf{S}_{2}\right) q\left(\mathbf{S}_{2}, \mathbf{S}_{2}^{*}\right)}
$$

onde $\pi($.$) é a distribuição condicional completa a posteriori de r_{2} \mathrm{e} w$,

$$
\begin{aligned}
\pi\left(\mathbf{S}_{2}\right) & =p\left(\tau_{2}, \mathbf{w} \mid \mathbf{y}, \boldsymbol{\beta}, \boldsymbol{\gamma}, \sigma^{2}, \phi_{2}\right) \\
& \propto\left[\prod_{i=1}^{m} w_{i}^{n_{i}}\right]^{1 / 2} \phi_{2}^{\left(\frac{m-\sum w_{i}}{1-\tau_{2}}\right)}\left(1-\phi_{2}\right)^{\left(\frac{\sum w_{i}-m \tau_{2}}{1-\tau_{2}}\right)} \\
& \times \exp \left\{-\frac{1}{2 \sigma^{2}} \sum_{i=1}^{m} w_{i} \delta_{i}^{\prime} \delta_{i}\right\}
\end{aligned}
$$

e $q($.$) , a densidade geradora de candidatos, também Beta [a, b]$, como anteriormente. 


\section{EXEMPLOS DE APLICAÇÃO}

Para se ilustrar a metodologia de modelos lineares mistos robustos discutida no Capítulo 3, são utilizados dois exemplos experimentais nos quais se observam valores discrepantes em nível de resíduos e/ou nível de outros efeitos aleatórios.

Num primeiro exemplo, objetiva-se a comparação da distribuição $t$, distribuição slash e distribuição normal contaminada para os resíduos como alternativas robustas para a distribuição normal, bem como a comparação de modelos assumindo-se estas distribuições como univariadas ou multivariadas para indivíduos sob mesmo grupo de efeitos aleatórios.

Ainda com este exemplo é discutida a utilização das distribuições nor$\mathrm{mal} /$ independentes na deteç̧ão de valores discrepantes.

Num segundo exemplo, a distribuição robusta é considerada também para os efeitos aleatórios do modelo. Neste caso, objetiva-se a utilização e comparação de diferentes modelos $t$ e gaussianos.

\subsection{Estudo reprodutivo em ratas}

\subsubsection{Procedimentos metodológicos}

Como um primeiro exemplo ilustrativo de aplicação da metodologia de modelos lineares mistos robustos é considerado o conjunto de dados apresentado por Dempster et al. (1984), e reanalisado por Davidian \& Giltinan (1995), o qual se refere ao peso ao nascimento (gramas) de filhotes num estudo toxicológico em reprodução de ratas.

Neste experimento, dez fêmeas foram alocadas aleatoriamente em cada um de três tratamentos (controle, baixa e alta dose da substância teste), sendo que no grupo de alta dose foram obtidos dados somente de sete ninhadas.

É fácil perceber neste caso que, para cada filhote, existem duas fontes aleatórias de variação: variabilidade de filhote para filhote (dentro de ninhada) e variabilidade de ninhada para ninhada. 
O modelo adotado pelos autores para o estudo do efeito (fixo) de grupo experimental também incluiu tamanho de ninhada e sexo do fllhote como covariáveis adicionais (Dempster et al., 1984), e pode ser representado por (Davidian \& Giltinan, 1995)

$$
y_{i j}=\tau_{k}+l_{i} \lambda+x_{i j} \delta+b_{i}+e_{i j}
$$

onde $y_{i j}$ é o peso ao nascimento do $j$-ésimo filhote da $i$-ésima ninhada, $\tau_{k}$ representa a média do $k$-ésimo grupo experimetal, e $b_{i}$ é o efeito aleatório de ninhada. $l_{i}$ é o tamanho da $i$-ésima ninhada e $x_{i j}$ é uma variável indicadora assumindo o valor 0 para fêmeas e 1 para machos, de maneira que $\delta$ representa um termo para o efeito (fixo) de sexo e $\lambda$ é um coeficiente de regressão para a covariável tamanho da ninhada, assumida como constante para todos os grupos experimentais e para ambos os sexos.

Davidian \& Giltinan (1995), entretanto, alertam para o fato de que a utilização de tamanho de ninhada como covariável pode não ser muito apropriada uma vez que esta pode também ser influenciada pelo efeito de tratamentos.

Os resultados apresentados por Dempster et al. (1984) e Davidian \& Giltinan (1995) são apresentados na Tabela 1.

Tabela 1: Estimativas dos componentes de variância e de alguns contrastes e parâmetros de interesse para o conjunto de dados de peso ao nascimento de ratos.

\begin{tabular}{lcc}
\hline \hline Parâmetro & Estimativa & Erro Padrão \\
\hline Coeficiente para tamanho de ninhada $(\lambda)$ & $-0,129$ & 0,019 \\
Efeito de sexo $(\delta)$ & 0,359 & 0,047 \\
Efeito de tratamento: & & \\
$\quad$ Baixa dose - Controle & $-0,429$ & 0,150 \\
$\quad$ Alta dose - Controle & $-0,859$ & 0,182 \\
Componentes de variância: & & \\
$\quad$ Entre ninhadas $\sigma_{b}^{2}$ & 0,097 & \\
$\quad$ Dentro de ninhadas $\sigma_{e}^{2}$ & 0,163 & \\
\hline \hline
\end{tabular}


Um estudo dos resíduos estimados, entretando, denota alguns valores discrepantes, o que pode ser verificado num gráfico de resíduos padronizados (Figura 1), sugerindo que o modelo gaussiano não se ajusta bem ao conjunto global dos dados, e que provavelmente uma distribuição leptocúrtica seria mais adequada para esta situação.

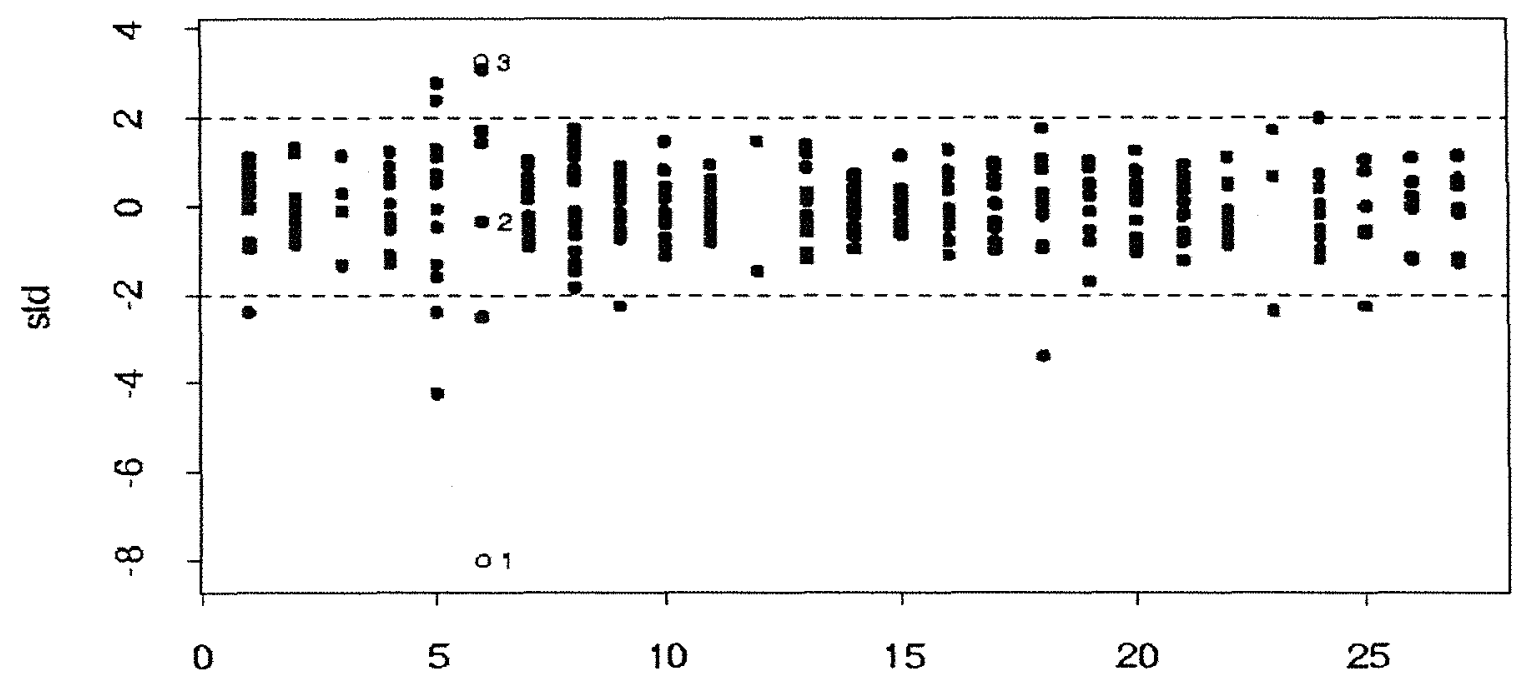

Ninhaqa

Figura 1: Resíduos padronizados de cada indivíduo, em cada ninhada, para o modelo gaussiano (Os números 1, 2 e 3 denotam animais que serão utilizados em discussão posterior com relação à detecção de valores discrepantes).

As três distribuições normal/independentes discutidas (distribuições $t$ de Student, slash e normal contaminada) serão aplicadas a este conjunto de dados. Para compará-las com o modelo normal, entretanto, os dados serão reanalisados dentro de um contexto bayesiano, assumindo-se ainda a distribuição gaussiana. Numa segunda etapa, a distribuição gaussiana dos resíduos será substituída pelas distribuições normal/independentes em duas maneiras diferentes.

No primeiro caso, será assumido que os vetores de resíduos relacionados a filhotes de uma mesma ninhada, $\mathbf{e}_{i}$, têm distribuições normal/independentes multivariadas independentes. 
Numa segunda alternativa, será assumido que os resíduos individuais de cada filhote, $e_{i j}$, apresentam distribuições normal/independentes univariadas e independentes.

Neste sentido, pela primeira situação considerada, uma alta variabilidade dentro de ninhada é penalizada, enquanto que na segunda somente indivíduos com resíduos discrepantes são penalizados.

O grupo de modelos com distribuições residuais multivariadas deve-se apresentar como uma alternativa interessante numa situação na qual, por exemplo, acredita-se que qualquer observação discrepante numa ninhada pode comprometer todos os indivíduos da mesma. Estatisticamente, o grupo de modelos com distribuições residuais univariadas assume total independência entre quaisquer $e_{i j}$, enquanto aqueles com distribuições multivariadas, apesar dos $e_{i j}$ de uma mesma ninhada serem não correlacionados, eles não são independentes.

Distribuições a priori uniformes (mas próprias) foram adotadas para os efeitos fixos e componentes de variância para todos os modelos. Para os parâmetros $\nu$, específicos para cada caso, as distribuições a priori foram definidas como no ítem 3.4 .

Através de inspeção gráfica e do método de diagnóstico de Raftery e Lewis (Raftery \& Lewis, 1992), foi adotado um periodo de descarte de 30 mil iterações mais 200 mil iterações adicionais com intervalos de sub-amostragem de 10 iterações para a cadeia de Gibbs obtida utilizando-se o programa CODA (Best et al., 1995).

Toda a análise dos valores obtidos do Amostrador de Gibbs e a apresentação gráfica da mesma foram realizadas utilizando-se o procedimento IML do SAS (SAS, 1989) e S-PLUS (Venables \& Ripley, 1996), respectivamente.

\subsubsection{Resultados e discussão}

Como referido anteriormente, o conjunto de dados de Dempster et al. (1984) foi inicialmente reanalisado dentro de um contexto bayesiano, utilizando-se o mesmo modelo proposto pelos autores e assumindo-se também a distribuição normal. 
A média, a mediana, o desvio padrão e alguns percentis da distribuição marginal a posteriori dos contrastes e parâmetros discutidos por Dempster et al. (1984) são apresentados na Tabela 2. Os resultados obtidos são bastante próximos daqueles encontrados pelo método de máxima verossimilhança, apresentados na Tabela 1.

Tabela 2: Média, mediana, desvio padrão (DP) e percentis $(2,5$ e $97,5 \%)$ das distribuições a posteriori marginais de alguns contrastes e parâmetros de interesse para o conjunto de dados de peso ao nascimento de ratos.

\begin{tabular}{lccccc}
\hline \hline Parâmetro & Média & Mediana & $\mathrm{DP}$ & $\mathrm{P}_{2,5}$ & $\mathrm{P}_{97,5}$ \\
\hline Coeficiente para tamanho de & & & & & \\
ninhada $(\lambda)$ & $-0,129$ & $-0,129$ & 0,020 & $-0,168$ & $-0,088$ \\
Efeito de sexo $(\delta)$ & 0,360 & 0,360 & 0,048 & 0,266 & 0,455 \\
Efeito de tratamento: & & & & & \\
$\quad$ Baixa dose - Controle & $-0,429$ & $-0,428$ & 0,164 & $-0,757$ & $-0,101$ \\
$\quad$ Alta dose - Controle & $-0,858$ & $-0,860$ & 0,199 & $-1,240$ & $-0,459$ \\
Componentes de variância: & & & & & \\
$\quad$ Entre ninhadas $\left(\sigma_{b}^{2}\right)$ & 0,121 & 0,112 & 0,048 & 0,058 & 0,239 \\
$\quad$ Dentro de ninhadas $\left(\sigma_{e}^{2}\right)$ & 0,165 & 0,165 & 0,014 & 0,141 & 0,194 \\
\hline \hline
\end{tabular}

Com o modelo gaussiano, uma vez que covariância igual a zero implica em independência, não existe diferença entre as duas situações de distribuições residuais discutidas anteriormente, e que serão aplicadas com as demais distribuições robustas.

É interessante notar que a distribuição normal é um caso particular das distribuições normal/independentes, correspondendo às distribuições $t$ ou slash com número infinito de graus de liberdade ou parâmetro $\nu$, respectivamente, ou a uma distribuição normal contaminada na qual a proporção de contaminates é nula.

Como discutido anteriormente, os resíduos estimados utilizando-se a 
distribuição normal sugerem que este modelo não se adequa muito bem a todo o conjunto de dados, e assim as três distribuições robustas apresentadas no ítem 3.4 serão utilisadas alternativamente para se reanalisar estes dados.

$\mathrm{Na}$ Figura 2, as densidades marginais a posteriori dos parâmetros $u$, estimadas usando-se a estimação pelo método de núcleo do S-PLUS (Venables \& Ripley, 1996), são apresentadas para as situações uni e multivariadas anteriormente discutidas. Estas densidades, para as distribuições $t$ e slash, são bastante concentradas ao redor de valores pequenos sugerindo, uma vez mais, a inadequabilidade do modelo gaussiano na análise destes dados.

O mesmo resultado foi obtido com a distribuição normal contaminada. Nota-se na Figura 2 que, apesar da pequena proporção de valores discrepantes (contaminantes), a variância da distribuição desta população é bem maior do que da população não contaminante.

Uma outra maneira de se verificar o melhor ajuste das distribuições leptocúrticas para este conjunto de dados é utilizando-se o Fator de Bayes (Kass \& Raftery, 1995).

As densidades marginais dos dados para cada modelo considerado foram obtidas utilizando-se a média harmônica da verossimilhança avaliada em cada valor simulado da cadeia de Gibbs (Newton \& Raftery, 1994), e são apresentadas na Tabela 3.

Tabela 3: Densidade de probabilidade marginal dos dados para cada um dos modelos considerados, $p(y \mid$ Modelo $)$.

\begin{tabular}{lcc}
\hline \hline \multirow{2}{*}{ Distribuição } & \multicolumn{2}{c}{ Modelo } \\
\cline { 2 - 3 } Gaussiana & Multivariado & Univariado \\
\hline de Student & $8,474 \times 10^{-83}$ & $8,474 \times 10^{-83}$ \\
Slash & $7,898 \times 10^{-73}$ & $7,181 \times 10^{-58}$ \\
Normal Contaminada & $6,190 \times 10^{-79}$ & $5,628 \times 10^{-62}$ \\
\hline \hline
\end{tabular}



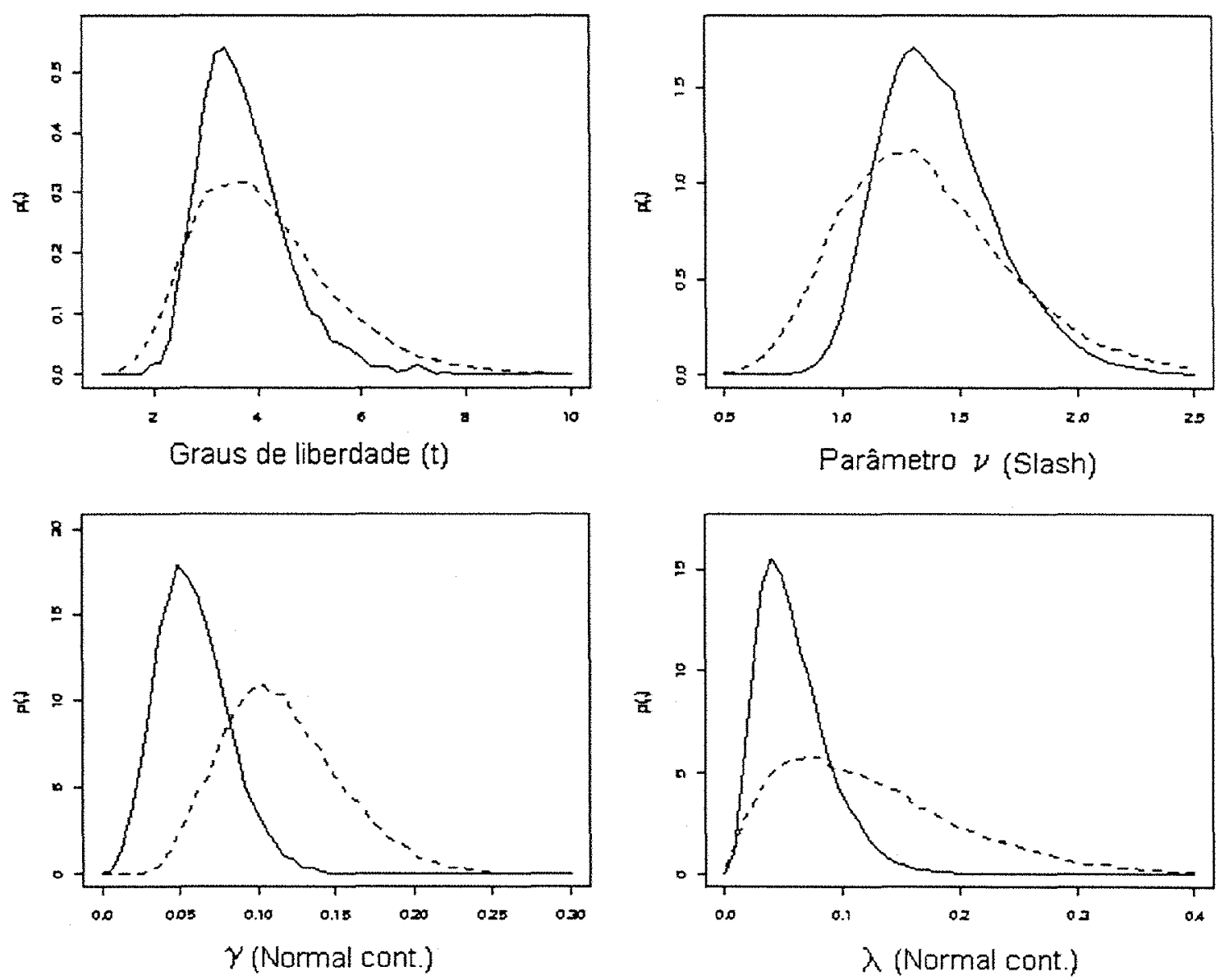

Figura 2: Densidade marginal a posteriori dos parâmetros $\nu$ de cada distribuição considerada, para as situações multivariadas (linhas segmentadas) e univariadas (linhas contínuas).

Os valores de Fatores de Bayes de aproximadamente $9,32 \times 10^{9}$; $2,64 \times 10^{15}$ e 7304,2 ; respectivamente, para as distribuições $t$, slash e normal contaminada, quando comparadas com o modelo gaussiano, denotam a inadequabilidade deste último.

Mais um resultado interessante é que todos os modelos normal/independentes univariados foram sensivelmente superiores aos seus respectivos multivariados, com valores de Fator de Bayes de $9,09 \times 10^{14} ; 1,64 \times 10^{10}$ e $9,09 \times 10^{16}$, respectivamente, para as distribuições $t$, slash e normal contaminada. 
Entre estes modelos univariados, o melhor ajuste foi apresentado pelo modelo empregando a distribuição slash, com valores para o Fator de Bayes de 5,11 e $6,52 \times 10^{4}$ quando comparado com os modelos com distribuições $t$ e normal contaminada, respectivamente.

Além dos modelos robustos apresentarem ajustes bem mais consistentes do que o modelo gaussiano para este conjunto de dados, são verificadas também algumas diferenças nas estimativas dos parâmetros e contrastes de interesse, quando comparadas às do modelo normal.

Como exemplo, na Figura 3 é apresentado um gráfico "box plot" das amostras a posteriori da diferença de pesos entre machos e fêmeas para os sete modelos considerados neste estudo. Pode ser verificado que o modelo normal superestima um pouco esta diferença, e com um variância maior também.

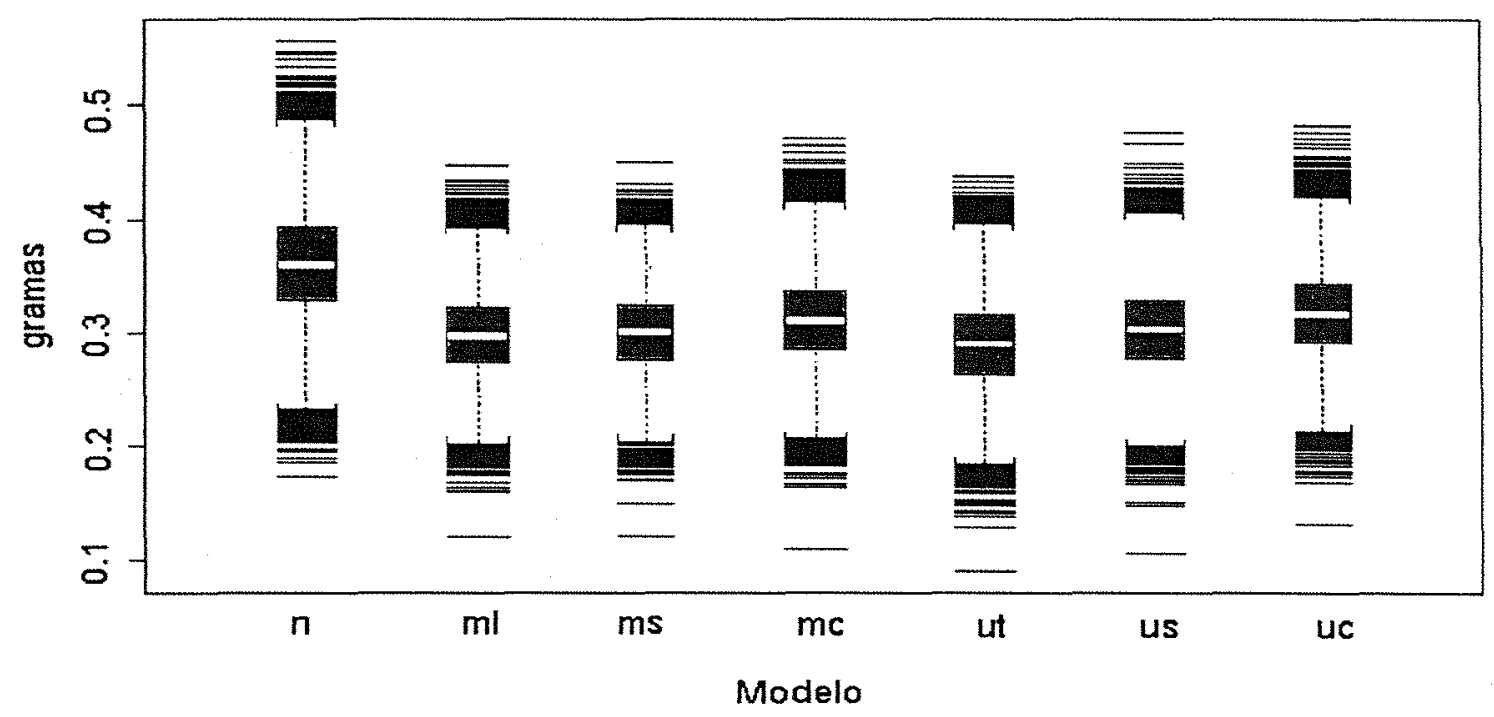

Figura 3: Gráfico "box plot" dos valores obtidos pelo Amostrador de Gibbs para o contraste entre pesos de machos e fêmeas, para cada um dos modelos considerados (n: normal: m: multivariada; u: univariada; t: $t$ de Student; s: slash; e c: normal contaminada).

Os modelos normal/independentes uni e multivariados não apresentaram diferenças importantes com relação a este contraste. 
Uma outra interessante observação com relação às distribuições normal/independentes é que elas podem ser utilizadas não só para estimação robusta, mas também para a deteç̧ão de valores discrepantes no conjunto de dados.

$\mathrm{Na}$ Figura 4 são apresentadas as distribuições marginais a posteriori das variáveis ponderadoras $\left(w_{i j}\right)$ relativas a cada uma das distribuições normal/independentes para os três filhotes da ninhada de número seis assinalados na Figura 1.
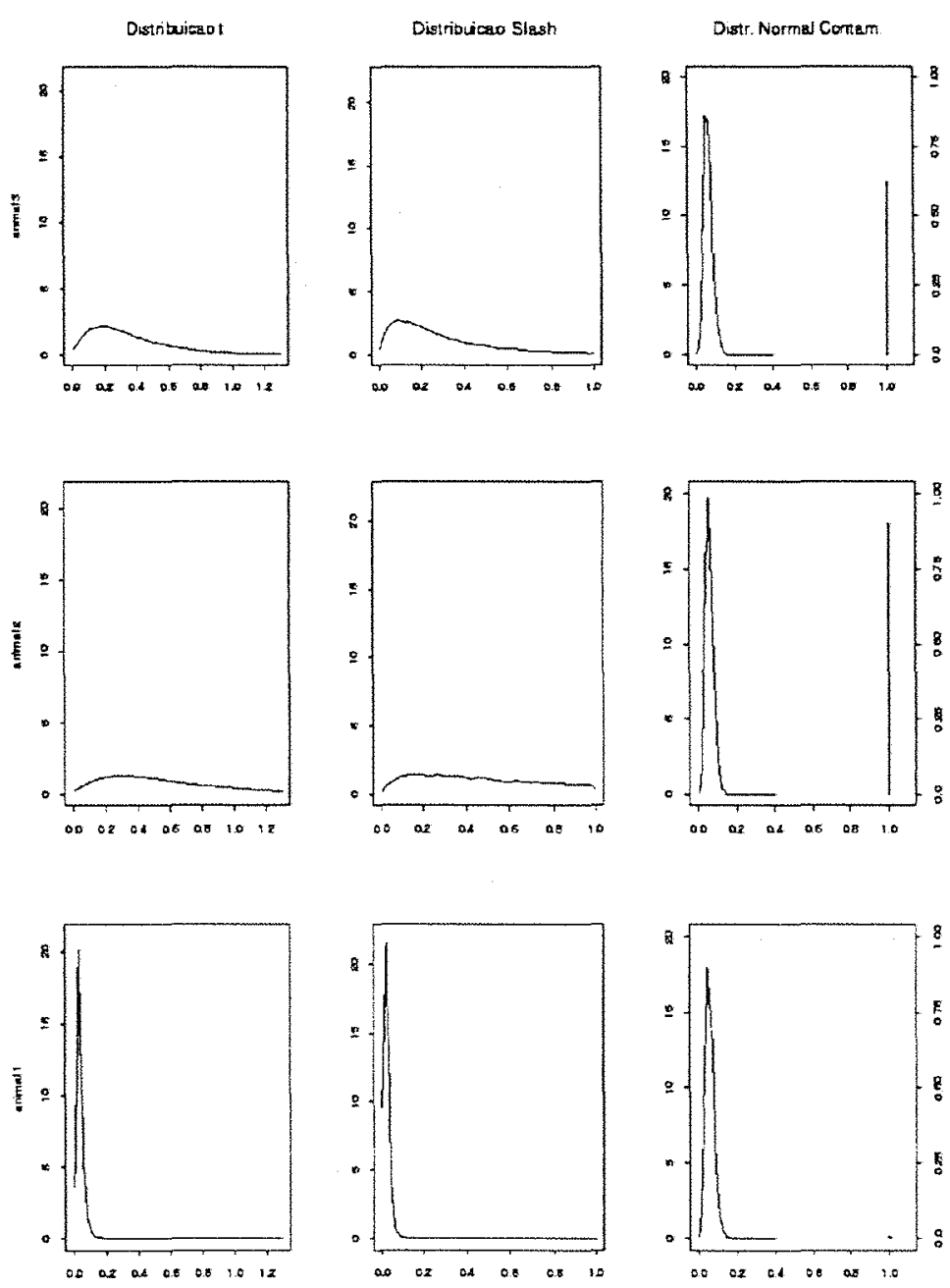

Figura 4: Distribuições marginais a posteriori das variáveis ponderadoras dos indivíduos 1, 2 e 3 assinalados na Figura 1, para as distribuições normal/independentes consideradas ( $\mathrm{O}$ eixo adicional à direita refere-se à probabilidade de $w_{i j}=1$ ). 
Observando-se a Figura 4, é fácil se notar que quanto maior o resíduo estimado para cada indivíduo, menor é o valor assumido para sua respectiva variável ponderadora. A mesma estratégia pode ser utilizada com as distribuições multivariadas, mas na detecção de altas variabilidades dentro de ninhadas.

\subsection{Estudo ortodôntico em crianças}

\subsubsection{Procedimentos metodológicos}

Como mais uma aplicação da metodologia de modelos lineares mistos robustos, um exemplo com dados longitudinais com alguns valores discrepantes tanto em nível de efeitos aleatórios quanto em de resíduos é utilizado para comparar o desempenho de alguns modelos gaussianos e modelos $t$.

Os dados aqui considerados, apresentados inicialmente por Potthoff \& Roy (1964), e mais recentemente rediscutidos por Verbeke \& Molenberghs (1997), comparando alguns modelos gaussianos, e por Pinheiro et al. (1998), utilizando um modelo robusto no qual emprega a distribuição $t$ para os efeitos aleatórios e resíduos, referem-se a um estudo ortodôntico com 16 meninos e 11 meninas com idades entre 8 e 14 anos.

A variável resposta é a distância (milímetros) entre a pituitária e a fissura pterigomaxilar, a qual foi medida aos $8,10,12$ e 14 anos de idade de cada criança. Os dados podem ser obtidos em Potthof \& Roy (1964) e Verbeke \& Molenberghs (1997), e são apresentados na Figura 5, juntamente com as regressões lineares de mínimos quadrados para cada criança.

O estudo inicia-se pela utilização e comparação de alguns modelos lineares mistos gaussianos propostos por Verbeke \& Molenberghs (1997) e Pinheiro et al.(1998), mas numa perspectiva bayesiana. Uma discussão detalhada acerca da adequacidade dos modelos lineares mistos para a análise deste conjunto de dados é apresentada em ambas as referências acima citadas. 


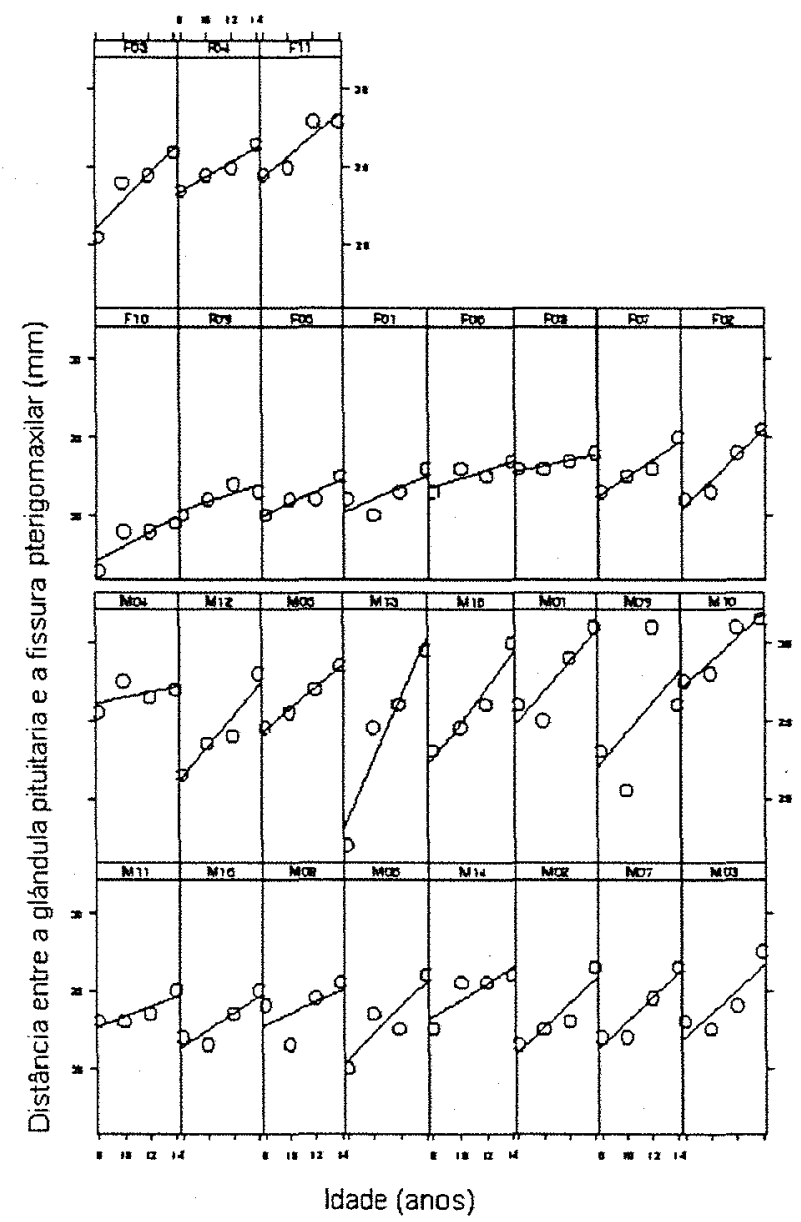

Figura 5: Regressões de mínimos quadrados da distância $(\mathrm{mm})$ entre a pituitária e a fissura pterigomaxilar em função da idade (anos), para os 16 meninos e 11 meninas do estudo ortodôntico (Adaptado de Pinheiro et al. (1998)).

O modelo linear misto geral para este exemplo pode ser expresso como

$$
\mathrm{y}_{i j}=X_{i} \theta+Z \mathrm{~b}_{i j}+\mathrm{e}_{i j}
$$

onde $i=1,2$, representa $o$ índice para meninos e meninas, respectivamente, e $j=1, \ldots, m_{i}$, representa os indivíduos de cada sexo, onde $m_{1}=16$ e $m_{2}=11$. Todas as definições são as mesmas do modelo (1) discutido no ítem 2.1.1.

Os vetores de efeitos "fixos" e "aleatórios" foram definidos como $\boldsymbol{\theta}=\left(\alpha_{1}, \alpha_{2}-\alpha_{1}, \beta_{1}, \beta_{2}-\beta_{1}\right)^{\prime}$ e $\mathbf{b}_{i j}=\left(\alpha_{i j}, \beta_{i j}\right)^{\prime}$, e as respectivas matrizes de in- 
cidência, como

$$
X_{i}=\left[\begin{array}{cccc}
1 & i-1 & 8 & 8(i-1) \\
1 & i-1 & 10 & 10(i-1) \\
1 & i-1 & 12 & 12(i-1) \\
1 & i-1 & 14 & 14(i-1)
\end{array}\right] \text { e } \quad Z=\left[\begin{array}{cc}
1 & 8 \\
1 & 10 \\
1 & 12 \\
1 & 14
\end{array}\right]
$$

Quatro casos específicos do modelo geral (10) foram considerados. O primeiro foi discutido por Verbeke \& Molenberghs (1997), denotado como Modelo 6 , no qual foi assumida uma variância residual comum para meninos e meninas, i.e., $\operatorname{Var}\left(\mathbf{e}_{i j}\right)=\sigma^{2} I_{(4)}$, tendo-se interceptos e coeficientes de regressão como aleatórios.

Um segundo modelo, denotado por Modelo 7 pelos mesmos autores, tem somente os interceptos como aleatórios. Neste caso, os efeitos aleatórios são escalares, $b_{i j}=\alpha_{i j}$, e Z é um vetor unitário.

O terceiro modelo aqui considerado foi aquele estudado por Pinheiro et al. (1998), no qual novamente interceptos e regressores são assumidos como aleatórios, porém, diferentemente do Modelo 6 previamente descrito no sentido de que as variâncias residuais são consideradas diferentes para meninos e meninas, i.e., $\operatorname{Var}\left(\mathbf{e}_{i j}\right)=\sigma_{i}^{2} I_{(4)}$.

Para completar, considerou-se mais um modelo gaussiano, aquele com somente os interceptos como aleatórios, mas com distintas variâncias residuais para cada sexo.

$\mathrm{Na}$ Tabela 4 é apresentado um resumo das características principais de cada um dos modelos descritos acima, os quais serão designados como modelos Normal 1, Normal 2, Normal 3 e Normal 4, respectivamente.

As mesmas distribuições uniformes a priori foram assumidas para os efeitos "fixos" e parâmetros de dispersão para todos os modelos.

O Amostrador de Gibbs foi novamente utilizado na condução da análise a posteriori utilizando-se uma cadeia única com 40 mil iterações descartadas para a convergência, mais 440 mil iterações adicionais com intervalos de espaçamento de 20 iterações, resultando numa amostra efetiva de 22 mil valores obtidos para a estimação de densidades marginais a posteriori e demais estatísticas de interesse. 
Tabela 4: Caracterização dos modelos lineares gaussianos utilizados no estudo ortodôntico em crianças.

\begin{tabular}{lcc}
\hline \hline Modelo & Efeitos Aleatórios & Variâncias Residuais \\
\hline Normal 1 & interceptos e regressores & iguais \\
Normal 2 & somente interceptos & iguais \\
Normal 3 & interceptos e regressores & diferentes \\
Normal 4 & somente interceptos & diferentes \\
\hline \hline
\end{tabular}

A comparação dos modelos foi efetuada usando-se o Fator de Bayes, calculado pelo método da média harmônica da verossimilhança avaliada em cada um dos pontos amostrados na cadeia de Gibbs (Newton \& Raftery, 1994).

\subsubsection{Resultados e discussão}

As densidades a posteriori marginais dos dados para cada um dos modelos gaussianos considerados são apresentadas na Tabela 5.

Tabela 5: Densidade de probabilidade marginal dos dados para cada um dos modelos gaussianos considerados.

\begin{tabular}{lc}
\hline \hline Modelo & $p(y \mid$ Modelo $)$ \\
\hline Normal 1 & $4,037 \times 10^{-88}$ \\
Normal 2 & $2,022 \times 10^{-90}$ \\
Normal 3 & $4,227 \times 10^{-82}$ \\
Normal 4 & $2,266 \times 10^{-84}$ \\
\hline \hline
\end{tabular}

Verbeke \& Molenberghs (1997) discutem em seu trabalho a dificuldade da comparação dos Modelos 6 e 7 (os quais correspondem aos modelos aqui denominados Normal 1 e Normal 2, respectivamente) pelo Teste da Razão de Verossimilhança. 
Neste sentido, o Fator de Bayes mostra-se bastante flexível e útil para seleção de modelos e pode ser utilizado inclusive em situações com modelos não hierárquicos.

No trabalho destes autores, o Modelo 7 foi preferido para se analisar este conjunto de dados, resultado contrário ao obtido aqui pelo Fator de Bayes, com valor de 199,7; indicando, ainda que não expressivo, um melhor ajuste do modelo Normal 1 quando comparado com o modelo Normal 2, na análise deste conjunto de dados.

Também utilizando-se o Fator de Bayes, pode-se observar que os modelos com variâncias residuais diferentes para os dois sexos apresentaram ajustes substancialmente melhores do que seus correspondentes homocedásticos, assim como também os modelos com interceptos e regressores aleatórios quando comparados com aqueles com somente interceptos aleatórios.

Tendo em vista estes resultados, pode-se concluir que o modelo proposto por Pinheiro et al. (1998), denotado aqui como Normal 3, foi o melhor entre todos os modelos gaussianos considerados.

Como discutido no artigo destes autores e pode também ser verificado na Figura 5, o menino de número 13 (M13) apresenta estimativa de coeficiente de regressão consideravelmente maior, e o menino de número 9 (M09) apresenta maior variação ao redor da regressão ajustada em relação aos demais indivíduos. Estas observações sugerem que um modelo empregando uma distribuição de caudas longas (leptocúrtica) pode se apresentar como mais adequado para acomodar estes valores discrepantes.

Novamente, para esta situação, o Fator de Bayes pode ser utilizado para se comparar o ajuste de modelos robustos com os gaussianos.

Neste exemplo será utilizada a distribuição $t$ como uma alternativa robusta para a distribuição normal dos efeitos "aleatórios" e dos resíduos, mas obviamente outra distribuição normal/independente discutida anteriormente poderia ser preferida e utilizada de maneira similar.

A versão $t$ do modelo linear misto geral denotado como Normal 3, com interceptos e regressores aleatórios, e diferentes variâncias residuais para cada sexo, 
pode ser expressa como

$$
\mathrm{y}_{i j}=X_{i} \theta+Z \gamma_{i j}+\varepsilon_{i j}
$$

onde $\gamma_{i j}=u_{i j}^{-1 / 2} \mathbf{b}_{i j}$ e $\varepsilon_{i j}=w_{i j}^{-1 / 2} \mathbf{e}_{i j}$, onde $u_{i j}$ e $w_{i j}$ são variáveis gama, como discutido nos itens 2.4 .3 e 3.4.1. Todas as demais definições são as mesmas do modelo (11).

O primeiro modelo do grupo robusto estudado neste trabalho é aquele sugerido por Pinheiro et al. (1998), no qual considerou-se o mesmo valor para a variável ponderadora dos vetores de efeitos aleatórios e de resíduos de cada indivíduo, i.e., $\gamma_{i j}=w_{i j}^{-1 / 2} \mathbf{b}_{i j}$ e $\varepsilon_{i j}=w_{i j}^{-1 / 2} \mathbf{e}_{i j}$, onde $w_{i j} \sim \operatorname{Gama}\left(\nu_{i} / 2, \nu_{i} / 2\right)$, correspondendo a diferentes graus de liberdade para cada sexo. Para este modelo, denotado aqui por Robusto 1, foram adotadas as mesmas distribuições a priori utilizadas anteriormente com os modelos gaussianos, e para o número de graus de liberdade, $p\left(\nu_{i}\right) \propto \nu_{i}^{-2}$, $\nu_{i} \geq 1$, correspondendo a uma distribuição uniforme para seu inverso, como discutido no item 3.4.1.

A densidade marginal a posteriori estimada dos dados para o modelo Robusto 1 foi $9,476 \times 10^{-82}$, a qual não denota muita melhora no ajuste para este conjunto de dados quando comparado com a versão gaussiana, o modelo Normal 3, com um valor de Fator de Bayes estimado em 2,2.

Pelo método de máxima verossimilhança, as estimativas pontuais apresentadas por Pinheiro et al. (1998) para os graus de liberdade foram $\nu_{1}=5,784$ e $\nu_{2}=5,9 \times 10^{6}$. Usando inferência bayesiana, os intervalos centrais a posteriori a $95 \%$ de probabilidade obtidos foram $[1,55 ; 22,2]$ e $[1,73 ; 226,0]$, respectivamente para $\nu_{1}$ e $\nu_{2}$.

Pode-se verificar, portanto, que a distribuição marginal a posteriori para os graus de liberdade para as meninas é bastante menor do que a estimativa pontual obtida por máxima verossimilhança.

Uma outra análise bayesiana empregando-se o mesmo modelo Robusto 1, mas mudando as distribuições a priori para os graus de liberdade para distribuições uniformes com limites superiores para o domínio das mesmas, resultou em distribuições a posteriori "flat" para estes parâmetros. Isto revela que os dados 
não contêm muita informação sobre estes parâmetros e, em decorrência disto, as distribuições a priori terão uma grande influência nos resultados a posteriori.

Por este mesmo raciocínio, pode-se perceber também que aquelas estimativas pontuais obtidas por máxima verossimilhança apresentam bem pouca massa ao seu redor, o que resultará obviamente em intervalos de confiança de grande amplitude.

Como no presente trabalho não se tem interesse no estudo de diferentes distribuições a priori e sua influência nos resultados, mas sim comparar modelos alternativos para a estimação robusta para este conjunto de dados, foi adotada a distribuição a priori inicialmente utilizada como padrão para todos os modelos subsequentes.

Uma primeira alternativa ao modelo Robusto 1, sugerido Pinheiro et al. (1998), seria um modelo que permitisse diferentes pesos (valores para as variáveis ponderadoras) para os vetores de efeitos "aleatórios" e de resíduos de cada indivíduo. Esta alternativa parece bastante razoável uma vez que um vetor residual discrepante para qualquer criança não necessariamente corresponde a um vetor de efeitos "aleatórios" discrepante também, e vice-versa, de maneira que diferentes pesos irão tratá-los mais isoladamente.

Este segundo modelo, denotado por Robusto 2, corresponde ao modelo (12), onde tanto $u_{i j}$ quanto $w_{i j}$ têm distribuições gama com os mesmos parâmetros, i.e., $u_{i j}, w_{i j} \sim \operatorname{Gama}\left(\nu_{i} / 2, \nu_{i} / 2\right)$. O modelo Robusto 2 , apesar de se apresentar mais realista do que o modelo Robusto 1, ainda apresenta uma forte suposição e restrição que é a mesma distribuição para $u_{i j}$ e $w_{i j}$.

Sem esta restrição, um terceiro modelo é proposto, no qual distribuições gama diferentes são assumidas para os pesos dos vetores de efeitos aleatórios e de resíduos. Neste caso, entretanto, em decorrência do pequeno tamanho amostral e da dificuldade em se estimar os diferentes parâmetros para as distribuições dos efeitos aleatórios de cada sexo, é assumido que $u_{i j} \sim \operatorname{Gama}(\phi / 2, \phi / 2)$ e $w_{i j} \sim \operatorname{Gama}\left(\nu_{i} / 2, \nu_{i} / 2\right)$, o que corresponde a uma distribuição comum para todos os efeitos aleatórios, independentemente de sexo, mas diferentes daquelas relativas aos 
resíduos, as quais diferem entre si também. Este modelo será denominado Robusto 3.

Um último modelo, o qual não é um caso específico do modelo (11), mas uma situação ainda mais geral do que o modelo Robusto 3 , trata cada elemento do vetor de resíduo independentemente, como sugerido no trabalho de Rosa et al. (1998).

É interessante se notar que no modelo Robusto 3, os elementos de um mesmo vetor residual são não correlacionados mas não independentes, e um único valor discrepante dentro de um vetor compromete inteiramente o mesmo.

Neste último modelo, denotado por Robusto 4, cada elemento dos vetores residuais apresenta diferente peso, o qual pode ser expresso como $u_{i j} \sim \operatorname{Gama}(\phi / 2, \phi / 2)$ e $w_{i j k} \sim \operatorname{Gama}\left(\nu_{i} / 2, \nu_{i} / 2\right)$, onde $k=1,2,3,4$ corresponde a um índice para idade de mensuração $(8,10,12$, e 14 anos, respectivamente).

As densidades marginais a posteriori estimadas dos dados para cada um dos modelos robustos considerados são apresentadas na Tabela 6.

Tabela 6: Densidade de probabilidade marginal dos dados para cada um dos modelos $t$ considerados.

\begin{tabular}{ll}
\hline \hline Modelo & $p(y \mid$ Modelo $)$ \\
\hline Robusto 1 & $9,476 \times 10^{-82}$ \\
Robusto 2 & $4,561 \times 10^{-81}$ \\
Robusto 3 & $1,498 \times 10^{-79}$ \\
Robusto 4 & $1,775 \times 10^{-78}$ \\
\hline \hline
\end{tabular}

O modelo Robusto 4 apresentou ajuste consideravelmente melhor do que o modelo Normal 3, com Fator de Bayes de 4199,2; e foi o melhor entre todos os modelos robustos, com Fator de Bayes de 1873,4; 389,2 e 11,8; quando comparado com os modelos Robustos 1, 2 e 3, respectivamente.

$\mathrm{Na}$ Figura 6 são apresentadas as densidades marginais a posteriori 
estimadas para cada elemento do vetor de efeitos "fixos", utilizando-se os modelos Normal 3 e Robusto 4.
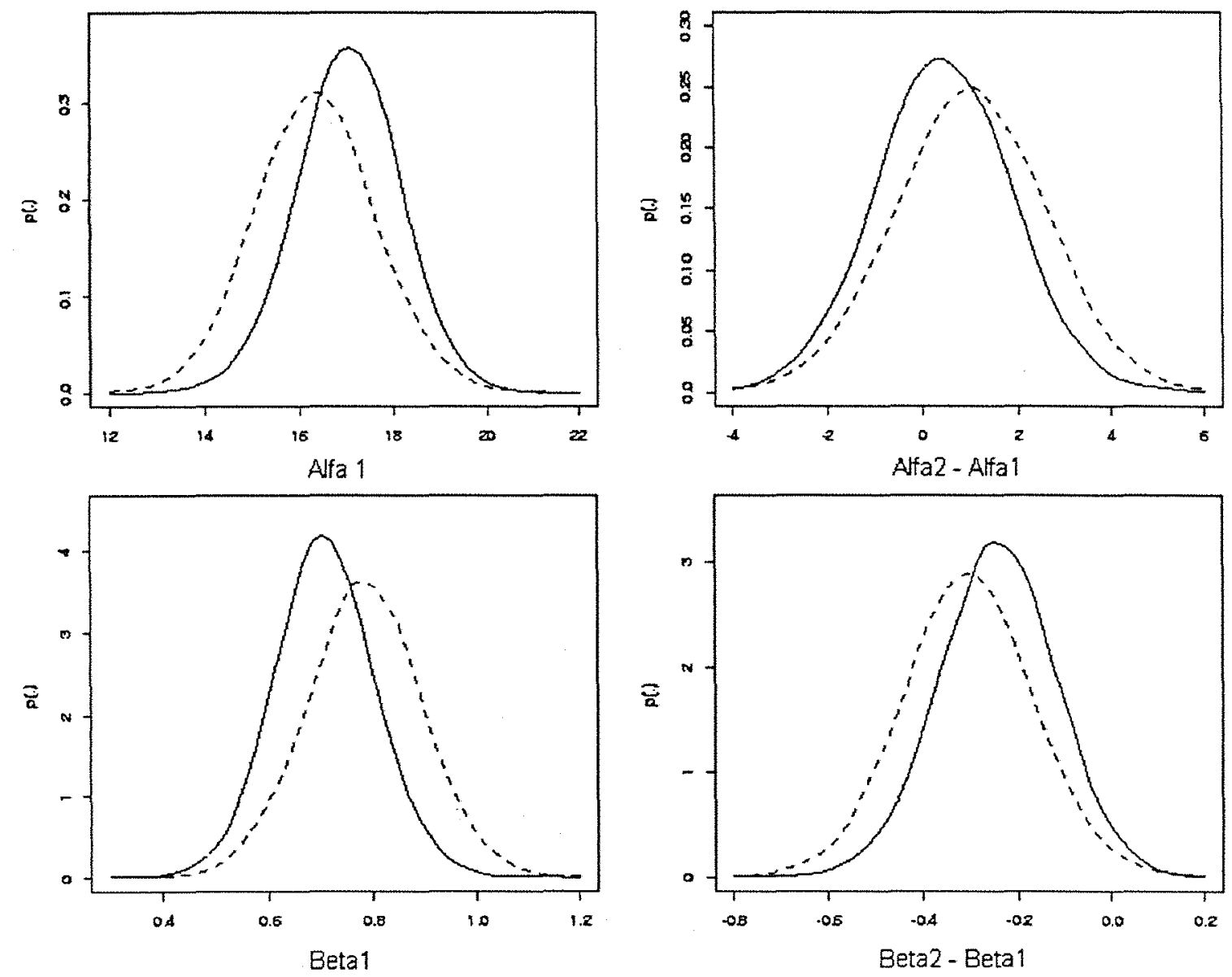

Figura 6: Densidades marginais a posteriori estimadas para cada elemento do vetor de efeitos "fixos" relativo ao estudo ortodôntico com crianças, considerando-se o modelo Normal 3 (linhas segmentadas) e o modelo Robusto 4 (linhas contínuas).

Pode-se observar que para cada um dos quatro parâmetros, as densidades são mais concentradas para o modelo robusto, correspondendo a intervalos centrais a posteriori mais estreitos, e que o modelo gaussiano está basicamente subestimando o intercepto, assim como superestimando o coeficiente de regressão, para o grupo de meninos. Estas estimativas estão sendo, provavelmente, bastante influenciadas pelas observações discrepantes do menino de número 13 (Figura 5). 
Com relação aos parâmetros de escala, a comparação entre eles não é direta porque estes não têm a mesma interpretação em cada um dos modelos.

Em geral, os parâmetros de dispersão não são de interesse principal, mas se este for o caso, podem-se obter as variâncias para o modelo $t$, a partir dos valores obtidos na Amostragem Gibbs, dadas por $\operatorname{Var}\left(\boldsymbol{\gamma}_{i j}\right)=[\delta /(\delta-2)] B$ e $\operatorname{Var}\left(e_{i j k}\right)=\left[\nu_{i} /\left(\nu_{i}-2\right)\right] \sigma_{i}^{2}$. Neste caso, entretanto, uma restrição ao espaço paramétrico se faz necessária uma vez que a variância de uma distribuição té definida somente para valores de graus de liberdade maiores do que 2 .

Os intervalos centrais a posteriori a $95 \%$ de probabilidade para os graus de liberdade, $[1,96 ; 161,0],[1,33 ; 9,78]$ e $[1,58 ; 102,0]$, respectivamente, para os efeitos aleatórios e para os resíduos de meninos e meninas no modelo Robusto 4, mostram que o principal problema com observações discrepantes neste estudo se deu em nível de resíduos, especificamente para os meninos, o qual apresentou um intervalo bastante estreito e concentrado em valores pequenos. 


\section{CONSIDERAÇÕES FINAIS}

As distribuições normal/independentes apresentam-se como um grupo de distribuições leptocúrticas bastante interessante para estimação robusta. Num contexto de modelos lineares mistos, estas distribuições podem ser utilizadas como alternativas à distribuição gaussiana tanto em nível de resíduos quanto em nível de efeitos aleatórios.

Trabalhando-se com modelagem bayesiana hierárquica, tem-se grande flexibilidade na utilização destas distribuiçães em modelos mistos como, por exemplo, assumindo-se distribuições normal/independentes distintas para resíduos e efeitos aleatórios.

A Amostragem Gibbs é de fácil implementação para a análise a posteriori das distribuições conjuntas obtidas. À excessão de alguns hiperparâmetros, como por exemplo, os graus de liberdade da distribuição t e o parâmetro $\tau$ da distribuição normal contaminada, todas as distribuições condicionais completas a posteriori apresentam forma fechada e portanto são de fácil simulação.

Nos casos em que não se tem uma distribuição condicional de forma fechada ou tem-se algum problema de irredutibilidade, o Algoritmo de MetropolisHastings pode ser utilizado alternativamente, assim como a Amostragem de Rejeição, no caso de distribuições unidimensionais.

De uma maneira geral, exceto em situações nas quais a suposição de normalidade do modelo amostral seja segura, uma distribuição normal/independente deve sempre ser preferida em relação à distribuição gaussiana, uma vez que esta última é um caso especial de qualquer destas distribuições robustas.

Uma outra interessante aplicação das distribuições normal/independentes, não só em situações de modelos lineares mistos, mas num contexto geral de aplicações estatísticas, refere-se à identificação de valores discrepantes, como foi discutido no exemplo 4.1.

Algumas sugestões para trabalhos futuros podem ser traçadas, como por exemplo: 
- Estudos de otimização da taxa de convergência da cadeia de Amostragem Gibbs, como apresentado por Chib \& Carlin (1997) em modelos lineares mistos gaussianos;

- Diferentes distribuições a priori para os diversos parâmetros do modelo, assim como diferentes métodos de amostragem das distribuições condicionais que não apresentam forma fechada;

- Inclusão de assimetria nas distribuições dos resíduos e demais fatores aleatórios do modelo;

Uma alternativa neste sentido, a qual não apresenta grandes dificuldades num contexto de inferência bayesiana, foi apresentada por Fernández \& Steel (1998), utilizando a distribuição $t$ em modelos de regressão linear.

A assimetria, como modelada por estes autores, implica na inclusão de somente um parâmetro adicional no modelo. Assim, se $p($.$) é qualquer função$ densidade de probabilidade, unimodal e simétrica em torno de 0 , pode-se gerar uma classe de distribuições assimétricas com um parâmetro adicional $\gamma \in(0, \infty)$ a partir de

$$
p(\varepsilon \mid \gamma)=\frac{2}{(\gamma+1 / \gamma)}\left\{p(\varepsilon \mid \gamma) I_{[0, \infty)}(\varepsilon)+p(\gamma \varepsilon) I_{(-\infty, 0)}(\varepsilon)\right\}
$$

onde $p(\varepsilon \mid \gamma)$ mantém a moda única no ponto 0 , mas perde sua simetria sempre que $\gamma \neq 1$, sendo assimétrica à esquerda se $0<\gamma<1$, e à direita se $\gamma>1$.

- Neste trabalho foram discutidas basicamente três das distribuições normal/independentes mais conhecidas. Outras alternativas podem ser estudadas e preferidas, como por exemplo a distribuição de Laplace (ou exponencial dupla), ou a distribuição logística (Andrews \& Mallows, 1974), ou ainda a distribuição estável (Lange \& Shinsheimer, 1993). 


\section{REFERÊNCIAS BIBLIOGRÁFICAS}

ANDREWS, D. F.; MALLOWS, C. L. Scale mixtures of normal distributions. Journal of the Royal Statistical Society, Series B. v.36, p.99-102, 1974.

BEST, N.; COWLES, M. K.; VINES, K. CODA: convergence diagnosis and output analysis software for Gibbs sampling output, Version $0.30,1995$.

CHIB, S.; CARLIN, B. P. On MCMC sampling in hierarchical longitudinal models. Statistical and Computing, 1998. /No prelo/

DAVIDIAN, M.; GILTINAN, D. M. Nonlinear models for repeated measurement data. New York: Chapman \& Hall, 1996. 359p.

DEMPSTER, A. P.; LAIRD, N. M.; RUBIN, D. B. Maximum likelihood from incomplete data via the EM algorithm. Journal of the Royal Statistical Society, Series B. v.39, p.1-22, 1977.

DEMPSTER, A. P.; LAIRD, N. M.; RUBIN, D. B. Iteratively reweighted least squares for linear regression when errors are normal/independent distributed. In: KRISHNAIAH, P. R. (Ed.) Multivariate analysis. Amsterdam: North-Holland, 1980, v.5, p.35-57.

DempsteR, A. P.; SELWYN, M. R.; PATEL, C. M.; ROTH, A. J.

Statistical and computational aspects of mixed model analysis. Applied Statistics, v.33, p.203-214, 1984.

FERNÀNDEZ, C.; STEEL, M. F. J. On Bayesian modeling of fat tails and skewness. Journal of the American Statistical Association, v.93, p.359$371,1998$. 
GELFAND, A. E.; RACINE-POON, A.; SMITH, A. F. M. Illustration of Bayesian inference in normal data models using Gibbs sampling. Journal of the American Statistical Association, v.85, n.412, p.972-985, 1990.

GELFAND, A. E.; SMITH, A. F. M. Sampling based approaches to calculating marginal densities. Journal of the American Statistical Association, v. 85, p.398-409, 1990.

GELMAN, A.; CARLIN, J. B.; STERN, H. S.; RUBIN, B. R. Bayesian data analysis. New York: Chapman \& Hall, 1995. 526p.

GEMAN, S.; GEMAN, D. Stochastic relaxation, Gibbs distributions and the Bayesian restoration of images. IEEE Transactions on Pattern Analysis and Machine Intelligence, v.6, p.721-741, 1984.

GEWEKE, J. Bayesian treatment of the independent Student- $t$ linear model. Journal of Applied Econometrics, v.8, p.S19-S40, 1993.

GILKS, W. R.; RICHARDSON, S.; SPIEGELHALTER, D. J. Markov chain Monte Carlo in practice. New York: Chapman \& Hall, 1996. 486p.

HARTLEY, H. O.; RAO, J. N. K. Maximum likelihood estimation for the mixed analysis of variance model. Biometrika, v.54, p.93-108, 1967.

HARVILLE, D. A. Maximum likelihood approaches to variance components estimation and related problems. Journal of the American Statistical Association, v.72, p.320-338, 1977.

HASTINGS, W. K. Monte Carlo sampling methods using Markov chains and their applications. Biometrika, v.57, p.97-109, 1970.

HENDERSON, C. R. Estimation of variance and covariance components. Biometrics, v.9, p.226-252, 1953.

HENDERSON, C. R. Applications of linear models in animal breeding. Guelph, Canada: University of Guelph, 1984. 
HOBERT, J. P.; CASELLA, G. The effect of improper priors on Gibbs sampling in hierarchical linear mixed models. Journal of the American Statistical Association, v.91, p.1461-1473, 1996.

KACKAR, R. N.; HARVILLE, D. A. Unbiasedness of two-stage estimation and prediction procedures for mixed linear models. Communications in Statistics - Theory and Methods, A10, p.1249-1261, 1981.

KASS, R. E.; RAFTERY, A. E. Bayes factors. Journal of the American Statistical Association, v.90, p.773-795, 1995.

LAMOTTE, L. R. A class of estimators of variance components. Lexington: University of Kentucky, Department of Statistics, 1970. (Technical Report, 10)

LAMOTTE, L. R. Locally best quadratic estimators of variance components. Lexington: University of Kentucky, Department of Statistics, 1971. (Technical Report, 22)

LAMOTTE, L. R. Quadratic estimation of variance components. Biometrics, v.29, p.311-330, 1973.

LAIRD, N. M.; WARE, J. H. Random-effects models for longitudinal data. Biometrics, v.38, p.963-974, 1982.

LANGE, K.; SINSHEIMER, J. S. Normal/independent distributions and their applications in robust regression. Journal of the American Statistical Association, v.2, p.175-198, 1993.

LANGE, K. L.; LITTLE, R. J. A.; TAYLOR, J. M. G. Robust statistical modeling using the $t$ distribution. Journal of the American Statistical Association, v.84, p.881-896, 1989.

LINDSTROM, M. J.; BATES, D. M. Newton-Raphson and EM algorithms for linear mixed effects models for repeated measures data. Journal of the 
American Statistical Association, v.83, p.1014-1022, 1988.

LITTLE, R. J. A. Robust estimation of the mean and covariance matrix from data with missing values. Applied Statistics, v.37, p.23-39, 1988.

LITTLE, R. J. A.; RUBIN, D. B. Statistical analysis with missing data. New York: John Wiley, 1987.

LIU, C. Missing data imputation using the multivariate $t$ distribution. Journal of Multivariate Analysis, v.53, p.139-158, 1995.

LIU, C. Bayesian robust multivariate linear regression with incomplete data. Journal of the American Statistical Association, v.91, p.1219-1227, 1996.

LIU, C. ML estimation of the multivariate $t$ distribution and the EM algoritms. Journal of the Multivariate Analysis, v.63, p.296-312, 1997.

LIU, C.; RUBIN, D. B. ML estimation of the $t$ distribution using EM and its extensions, ECM and ECME. Statistica Sinica, v.5, p.19-39, 1995.

MILLER, J. J. Asymptotic properties of maximum likelihood estimates in the mixed model of the analysis of variance. Annals of Statistics, v.5, p.746-762, 1977.

NEWTON, M. A.; RAFTERY, A. E. Approximate Bayesian inference by the weighted likelihood bootstrap (with discussion). Journal of the Royal Statistical Society, Series B. v.56, p.3-48, 1994.

PATTERSON, H. D.; THOMPSON, R. Recovery of interblock information when block sizes are unequal. Biometrika, v.58, p.545-554, 1971.

PINHEIRO, J. C.; LIU, C.; WU, Y. Robust estimation in linear mixed-effects models using the multivariate $t$-distribution. Biometrics, 1998. (submetido para publicação) 
POTTHOFF, R. F.; ROY, S. N. A generalized multivariate analysis of variance model useful especially for growth curve problems. Biometrika, v.51, p.313326,1964 .

RAFTERY, A. E.; LEWIS, S. M. How many iterations in the Gibbs sampler? In: BERNARDO, J. M.; BERGER, J. O.; DAVID, A. P.; SMITH, A. F. M. (Ed.) Bayesian statistics 4. Oxford: Oxford University Press, 1992. p.763-774.

RAO, C. R. Estimation of variance and covariance components - MINQUE theory. Journal of Multivariate Analysis, v.1, p.257-275, 1971a.

RAO, C. R. Minimum variance quadratic unbiased estimation of variance components. Journal of Multivariate Analysis, v.1, p.445-456, 1971b.

RIPLEY, B. D. Stochastic simulation. New York: Wiley, 1987.

ROGERS, W. H.; TUKEY, J. W. Understanding some long-tailed distributions. Statistica Neerlandia, v.26, p.211-226, 1972.

ROSA, G. J. M.; GIANOLA, D.; PADOVANI, C. R. Bayesian analysis of some robust mixed linear models with application to birth weight in mice. In: VALENCIA INTERNATIONAL MEETING ON BAYESIAN STATISTICS, 6., Las Fuentes, 1998.

RUBIN, D. B. Iteratively reweighted least squares. In: KOTZ, S.; JOHNSON, N. L.; READ, C. B. (Ed) Encyclopedia of the statistical sciences. New York: Wiley, 1983. v.4.

SAS INSTITUTE. SAS/IML Software: usage and reference, version 6. 1.ed. Cary, NC: SAS Institute Inc., 1989. 501p.

SEARLE, S. R.; CASELLA, G.; MCCULLOCH, C. E. Variance components. New York: Wiley, 1992. 
STRANDÉN, I. J. Robust mixed effects linear models with $t$ distributions and application to dairy cattle breeding. Madison, 1996. 176p. Thesis (Ph.D.) University of Wisconsin.

STRANDÉN, I.; GIANOLA, D. Gaussian versus student- $t$ mixed effects linear models for milk yield in Ayrshire cattle. In: ANNUAL MEETING OF THE EUROPEAN ASSOCIATION FOR ANIMAL PRODUCTION, 48., Vienna, 1997.

SUTRADHAR, B..C.; ALI, M. M. Estimation of the parameters of a regression model with a multivariate $t$ error variable. Communications in Statistics - Theory and Methods, v.15(2), p.429-450, 1986.

TANNER, M. A. Tools for statistical inference: methods for the exploration of posterior distributions and likelihood functions. 2.ed. New York: SpringerVerlag, 1993.

THOMPSON, W. A. The problem of negative estimates of variance components. Annals of Mathematical Statistics, v.33, p.273-289, 1962.

THOMPSON, W. A. Non-negative estimates of variance components. Technometrics, v.5, p.441-449, 1963.

VENABLES, W. N.; RIPLEY, B. D. Modern applied statistics with SPlus. New York: Springer-Verlag, 1996. 462p.

VERBEKE, G.; LESAFFRE, E. A linear mixed-effects model with heterogeneity in the random-effects population. Journal of the A merican Statistical Association, v.91, p.217-221, 1996.

VERBEKE, G.; MOLENBERGHS, G. Linear mixed models in practice. New York: Springer, 1997. 306p. 
WELSH, A. H.; RICHARDSON, A. M. Approaches to the robust estimation of mixed models. In: MADDALA, G. S.; RAO, C. R. (Ed.) Handbook of statistics. Elsevier, 1997. v.15, p.343-384.

WEST, M. Outliers models and prior distributions in Bayesian regression. Journal of the Royal Statistical Society, v.3, p.431-439, 1984.

ZEGER, S. L.; KARIM, M. R. Generalized linear models with random effects; A Gibbs sampling approach. Journal of the American Statistical Association, v.86, p.79-86, 1991.

ZELLNER, A. Bayesian and non-Bayesian analysis of the regression model with multivariate Student- $t$ error terms. Journal of the American Statistical Association, v.71, p.400-405, 1976. 